\title{
Magnetism and site exchange in CuFeAs and CuFeSb: A microscopic and theoretical investigation
}

\author{
Sirko Kamusella* and Hans-Henning Klauss \\ Institut für Festkörperphysik, Technische Universität Dresden, 01062 Dresden, Germany \\ Gohil S. Thakur, ${ }^{\dagger}$ Zeba Haque, and Laxmi C. Gupta \\ Department of Chemistry, Indian Institute of Technology, 110016 New Delhi, India \\ Ashok K. Ganguli \\ Department of Chemistry, Indian Institute of Technology, 110016 New Delhi, India \\ and Institute of Nano science and Technology (INST), Mohali, India (160062) \\ Inga Kraft, Ulrich Burkhardt, and Helge Rosner \\ Max Planck Institute for Chemical Physics of Solids, D-01087 Dresden, Germany
}

Hubertus Luetkens

Laboratory for Muon Spin Spectroscopy, Paul Scherrer Institut, CH-5232 Villigen PSI, Switzerland

Jeffrey W. Lynn

NIST Center for Neutron Research, National Institute of Standards and Technology, Gaithersburg, Maryland 20899-6102, USA

Yang Zhao

NIST Center for Neutron Research, National Institute of Standards and Technology, Gaithersburg, Maryland 20899-6102, USA

and Department of Materials Science and Engineering, University of Maryland, College Park, Maryland 20742, USA

(Received 15 November 2016; revised manuscript received 31 January 2017; published 13 March 2017)

\begin{abstract}
We have investigated the magnetic ground state of $\mathrm{CuFeAs}$ and $\mathrm{CuFeSb}$ by means of ${ }^{57} \mathrm{Fe}-\mathrm{Mössbauer}$ spectroscopy, muon spin rotation/relaxation $(\mu \mathrm{SR})$, neutron diffraction, and electronic structure calculations. Both materials share the 111-LiFeAs crystal structure and are closely related to the class of iron-based superconductors. In both materials there is a considerable occupancy of the $\mathrm{Cu}$ site by $\mathrm{Fe}$, which leads to ferromagnetic moments, which are magnetically strongly coupled to the regular Fe site magnetism. Our study shows that $\mathrm{CuFeAs}$ is close to an antiferromagnetic instability, whereas a ferromagnetic ground state is observed in CuFeSb, supporting theoretical models of anion height driven magnetism.
\end{abstract}

DOI: 10.1103/PhysRevB.95.094415

\section{INTRODUCTION}

The so-called "111" class of iron pnictides contains compounds which are isostructural to LiFeAs [1]. LiFeAs was the first discovered stoichiometric iron-based superconductor after LaFePO [2]. Interestingly, other parent compounds like $\mathrm{NaFeAs}$ [3] and LiFeP [4] are also superconducting, even though every single compound seems special. While LiFeP belongs [5] to the nodal class of iron-based phosphide superconductors [6], NaFeAs shows only a small superconducting volume fraction (but that rapidly increases upon electron doping $[7,8]$ ), along with the usual stripe antiferromagnetic (AFM) order and a nematic state in an exceptionally broad temperature range. In $\mathrm{LiFeAs}$ the details of the superconducting states are still under debate $[9,10]$. It features weak Fermi surface nesting conditions [11] and a moderate degree of electron correlation [12,13] and was initially considered

\footnotetext{
*sirko.kamusella@tu-dresden.de

${ }^{\dagger}$ Currently at the Max Planck Institute for Chemical Physics of Solids, 01187 Dresden, Germany.

${ }^{\ddagger}$ Visiting Scientist, Solid State Chemistry Lab., Dept. of Chemistry, IIT Delhi.
}

close to the stripe AFM ground state [14,15], but with a small interlayer spin coupling that prevents magnetic order [16]. LiFeAs is called a "paradigmatic testbed for theoretical models on multiband superconductors" [9].

Moon et al. predicted larger magnetic moments and stronger Hund's rule coupling in case of (As, Sb) substitution in the 111 class, stabilizing a magnetic phase [17]. According to their later work [18] the increased anion height $h_{\text {anion }}$ through larger ionic radius of $\mathrm{Sb}$ compared to As or P plays an important role, because an increasing anion height can render a magnetic system from the typically observed stripe over to double stripe AFM order (observed in FeTe [19]), to finally a ferromagnetic (FM) order for $h_{\text {anion }}>1.9 \AA$. This idea was further pursued by Yin, Lee, and $\mathrm{Ku}$ [20] to generate a unified picture of $h_{\text {anion }}$ driven magnetism in iron-based arsenides and chalcogenides in general. An increase in $h_{\text {anion }}$ corresponds to a decreased hopping of itinerant $d_{x z}$ or $d_{y z}$ electrons, consequently the increase in local moment.

The anion heights of LiFeP, NaFeAs, and LiFeAs increase from $1.32 \AA$ to $1.44 \AA$ and $1.51 \AA[1,3,4]$, respectively, and thus still range around the optimal anion height for superconductivity [21]. FeTe still exhibits the largest $h_{\text {anion }}=$ $1.74 \AA$ [22], showing bicolinear order. So far, ferromagnetism of the transition metal ions was only observed in Co based 
materials LaOCoP and LaOCoAs [23] until 2012, when $\mathrm{CuFeSb}$ was synthesized by Qian et al. [24]. CuFeSb is a soft ferromagnet, which shows a high spin polarization and a large anion height of $h_{\text {anion }}=1.84-1.89 \AA$ [24,25], vindicating the theories. Aside from the unique itinerant ferromagnetism of an iron pnictide in general, it is remarkable that a transition metal like $\mathrm{Cu}$ occupies the alkali ion position, opening an alternative route for the search of novel materials in the framework of ironbased superconductivity. This new route was consequently used for the synthesis of CuFeAs, where the substitution of Sb by As should naturally decrease $h_{\text {anion. }}$. However, the reported anion heights of 1.74 AA [26] to $1.8 \AA$ [27] are still large and justify a ground state with dominant ferromagnetic interactions and large magnetic moments. This was explicitly shown in electronic structure calculations applied to $\mathrm{CuFeAs}$ and $\mathrm{CuFeSb}$ [28]. Experimentally, these expectations are not reflected in $\mathrm{CuFeAs}$. Instead, an AFM ground state with $<0.2 \mu_{\mathrm{B}} / \mathrm{Fe}$ below $9 \mathrm{~K}$ by Thakur et al. [26] and a weak FM ground state with $0.4 \mu_{\mathrm{B}} / \mathrm{Fe}$ by Qian et al. [27] for $\mathrm{Cu}$ deficient specimens are observed.

The discrepancy of experimental observations and theoretical modeling challenges the intrinsic properties, structural parameters, and/or stoichiometry which have been reported so far. Both the investigations of Thakur [26] and Qian [27] give room for further interpretation because important statements are based on macroscopic susceptibility data, which show neither typical AFM nor typical FM behavior. The focus of our investigation is to examine both systems from a microscopic point of view by means of ${ }^{57} \mathrm{Fe}$ Mössbauer spectroscopy and muon spin rotation/relaxation $(\mu \mathrm{SR})$. Moreover, neutron diffraction was performed to determine the real crystallographic structure and search for and identify the CuFeAs long range order. Finally, DFT calculations quantify the effect of $\mathrm{Cu}$ deficiency on the stabilization of a magnetic ground state and anion height.

Our experiments on $\mathrm{CuFeSb}$ reproduce the soft FM order and evidence a strong coupling of the magnetic order parameters at the regular Fe site and secondary $\mathrm{Fe}$ site at the $\mathrm{Cu}$ position. One major result of this paper is that this $\mathrm{Fe}-\mathrm{Cu}$ mixing not only takes place in CuFeSb, but in CuFeAs as well. An FM behavior of CuFeAs thus can be attributed to the secondary Fe moments with a magnetic moment of $\approx 1 \mu_{\mathrm{B}}$ at the $\mathrm{Cu}$ site. The regular iron site on the other hand is close to a magnetic instability with saturated moments of $\approx 0.4 \mu_{\mathrm{B}}$. In our sample this magnetic ground state is only partially realized due to $\mathrm{Cu}$ deficiency or the doping effect of secondary iron. Because of the strong coupling of magnetic order parameters at both sites, there is a common ordering temperature of $\approx 11 \mathrm{~K}$.

\section{METHODS}

Polycrystalline specimens of $\mathrm{CuFeAs}$ and $\mathrm{CuFeSb}$ were prepared as described elsewhere [25,26]. For $\mu$ SR we used the same samples as previously described by Thakur et al. [26], whereas for neutron diffraction a new sample was synthesized. The samples are then accordingly designated as " $\mu \mathrm{SR}$ " and "neutron." With the exception of an increased homogeneity and slightly reduced amount of excess iron in the neutron sample, both samples are comparable with respect to Mössbauer and WDX analysis.
Mössbauer spectroscopy was performed in a standard transmission geometry employing a ${ }^{57} \mathrm{Co}$ source with initial activity of $2 \mathrm{GBq}$ and a source linewidth of $0.105 \mathrm{~mm} / \mathrm{s}$. A thin powder film was dried from an ethanol slurry and stabilized with dried sugar on top. The drive was run in sinusoidal mode minimizing the velocity error. The measurements were carried out in an Oxford continuous flow cryostat in under-pressure mode enabling temperatures from $2 \mathrm{~K}$ to $315 \mathrm{~K}$. The applied magnetic field has a homogeneity of $0.6 \%$ over the sample. Mössbauer spectra were analyzed with Moessfit [29] using transmission integral simulation, with the exception of those treated with maximum entropy method (MEM). Isomer shifts $\delta$ are given relatively to room temperature $\alpha$ iron.

$\mu$ SR measurements using a pressed pellet were carried out in the GPS instrument at the $\pi \mathrm{M} 3.2$ beamline of the Paul Scherrer Institute in Switzerland between $1.6 \mathrm{~K}$ and $290 \mathrm{~K}$ at zero and $5 \mathrm{mT}$ transverse field. The muon spin was rotated by $\approx 50^{\circ}$ with respect to the beam direction.

High intensity/coarse resolution neutron measurements at the NCNR were carried out on the BT-7 spectrometer using collimations of 80' full-width-at-half-maximum before and after the pyrolytic graphite (PG) monochromator and 80' radial collimator after the sample, with the 1-d position sensitive detector [30]. An incident monochromatic beam of $\lambda=2.4436 \AA$ was employed with a PG filter to suppress higher-order wavelengths. A closed cycle helium refrigerator was employed to take data at $2.5 \mathrm{~K}$ and $20 \mathrm{~K}$ to search for evidence of magnetic long range order. High resolution powder neutron diffraction data were collected on the BT-1 highresolution neutron powder diffractometer, using monochromatic neutrons of wavelength $\lambda=1.5403 \AA$ produced by a $\mathrm{Cu}(311)$ monochromator. Söller collimations before and after the monochromator and after the sample were 15', 20', and 7 ' full-width-at-half-maximum (FWHM), respectively. Data were collected in the $2 \theta$ range of $3^{\circ}$ to $168^{\circ}$ with a step size of $0.05 \theta$ at $20 \mathrm{~K}$, and structural refinements were performed using the FullProf program [31].

The homogeneity of the samples has been examined by wavelength dispersive $\mathrm{X}$-ray spectroscopy (WDX) on an electron microprobe Cameca SX100. The coarse powder of CuFeAs was mounted in a conductive epoxy resin with silver filler (EpoTek H20E), which allows us to prepare metallographic microstructures suitable for microprobe analyses. The local chemical composition of several particles was analyzed by using an electron beam of $20 \mathrm{kV}$ and $20 \mathrm{nA}$. The mass concentration of the elements was calculated from the intensities ratio of the $\mathrm{K}$ (alpha) lines measured on the CuFeAs phase and on the references $\mathrm{As}, \mathrm{Cu}$, and FeSi. The PAP model [32] was used to take matrix effects into account. Topographic effects do not influence the analytical results due to the planarity of the sample surface.

The electronic structure calculations for $\mathrm{CuFeAs}$ are performed using the full-potential local-orbital code (FPLO), version 9.07 [33,34]. The local (spin) density approximation [L(S)DA] with the Perdew and Wang flavor [35] of the exchange and correlation potential was selected. For the relaxation of the internal positions, also the generalized gradient approximation [36] was applied, yielding basically identical parameters. As lattice parameters we applied the neutron 
TABLE I. Neutron Rietveld refinement results of CuFeAs at 20 $\mathrm{K}$ according to Fig. 1 and previous XRD results [26]. $\mathrm{Cu}$ and $\mathrm{Fe}$ are indistinguishable by means of XRD. In neutron diffraction the ratio of both elements sensitively depends on the total site occupancy.

\begin{tabular}{|c|c|c|c|c|c|c|}
\hline & $\begin{array}{r}\text { Spac } \\
P 4\end{array}$ & $\begin{array}{l}\text { roup } \\
\mathrm{nm}\end{array}$ & \multicolumn{2}{|c|}{ Neutron diffr. } & \multicolumn{2}{|c|}{ XRD [26] } \\
\hline & \multicolumn{2}{|c|}{$a$} & \multicolumn{2}{|c|}{$3.7348(4) \AA$} & \multicolumn{2}{|c|}{$3.7442(2) \AA$} \\
\hline & \multicolumn{2}{|c|}{$c$} & \multicolumn{2}{|c|}{$5.8452(7) \AA$} & \multicolumn{2}{|c|}{$5.8925(4) \AA$} \\
\hline & $x$ & $y$ & $\mathrm{Z}_{\text {neutr. }}$ & Occ. neutr. & $\mathrm{Z}_{\mathrm{XRD}}$ & Occ.XRD \\
\hline $\mathrm{Fe}$ & 0.75 & 0.25 & 0 & $1.01(4)$ & 0 & $1.030(8)$ \\
\hline $\mathrm{Cu}$ & 0.25 & 0.25 & $0.6780(5)$ & $0.98(4)$ & $0.705(7)$ & $0.995(2)$ \\
\hline As & 0.25 & 0.25 & $0.2619(8)$ & 1 & $0.295(7)$ & $1.013(5)$ \\
\hline \multicolumn{3}{|c|}{ arsenic height $h_{A s}$ : } & \multicolumn{2}{|c|}{$1.531(1) \AA$} & \multicolumn{2}{|c|}{$1.74(4) \AA$} \\
\hline \multicolumn{3}{|c|}{ Fe bond angle $\alpha(2 \times)$ : } & \multicolumn{2}{|c|}{$101.3(2)^{\circ}$} & \multicolumn{2}{|c|}{$94(1)^{\circ}$} \\
\hline \multicolumn{3}{|c|}{ Fe bond angle $\beta(4 \times)$ : } & \multicolumn{2}{|c|}{$113.7(1)^{\circ}$} & \multicolumn{2}{|c|}{$118(1)^{\circ}$} \\
\hline
\end{tabular}

powder diffraction results listed in Table I. A $24 \times 24 \times 24$ (up to $32 \times 32 \times 32$ ) regular $k$-point mesh with resulting 1183 points in the irreducible part of the Brillouin zone was used to obtain well converged results with respect to the density of states (DOS) and the magnetic moments. To model the different $\mathrm{Cu}$ content continuously and to investigate the dependence of the electronic properties from this parameter, we applied the virtual crystal approximation (VCA) [37,38]. Uncertainties where indicated represent one standard deviation throughout the paper.

\section{RESULTS}

\section{A. Structural analysis using neutron diffraction on $\mathrm{CuFeAs}$}

A preliminary structural refinement of the BT-7 data indicated that both the $\mathrm{Cu}$ and $\mathrm{Fe}$ sites are fully occupied for the neutron sample. Subsequently high resolution data were collected at $20 \mathrm{~K}$ with a vanadium sample holder. Figure 1 shows the neutron powder diffraction pattern and Rietveld refinement fit results for $\mathrm{CuFeAs}$. The colored vertical lines show the Bragg positions of CuFeAs, of $18 \% \mathrm{Cu}_{3} \mathrm{As}$ (PDF\#741068), and of $8 \% \mathrm{Fe}_{3} \mathrm{O}_{4}$ [39]. The Rietveld refinement results for $\mathrm{CuFeAs}$ are listed in Table I. The results demonstrate that all sites are fully occupied consistent with previously reported X-ray results [26]. Presence of FeAs impurity can be excluded.

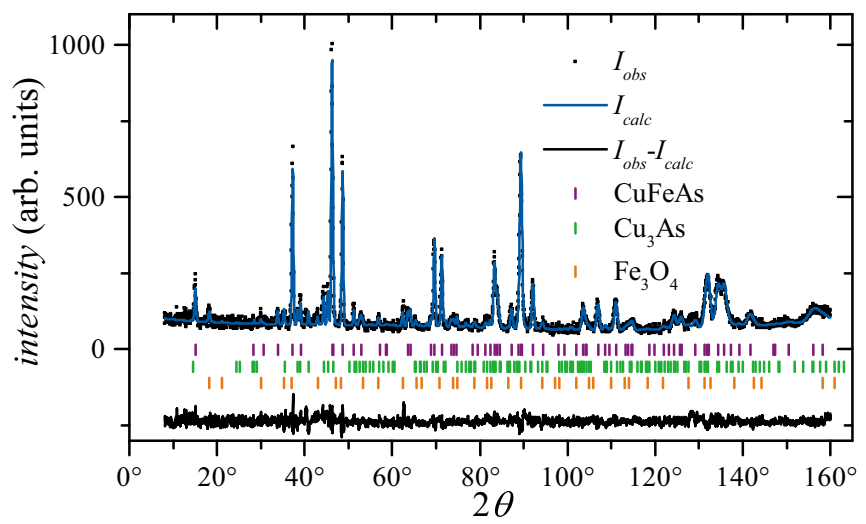

FIG. 1. Neutron powder diffraction pattern collected at $20 \mathrm{~K}$ (Bragg R-factor $10.26 \%$, RF-factor $12.6 \%, \chi^{2}=1.22$ ).

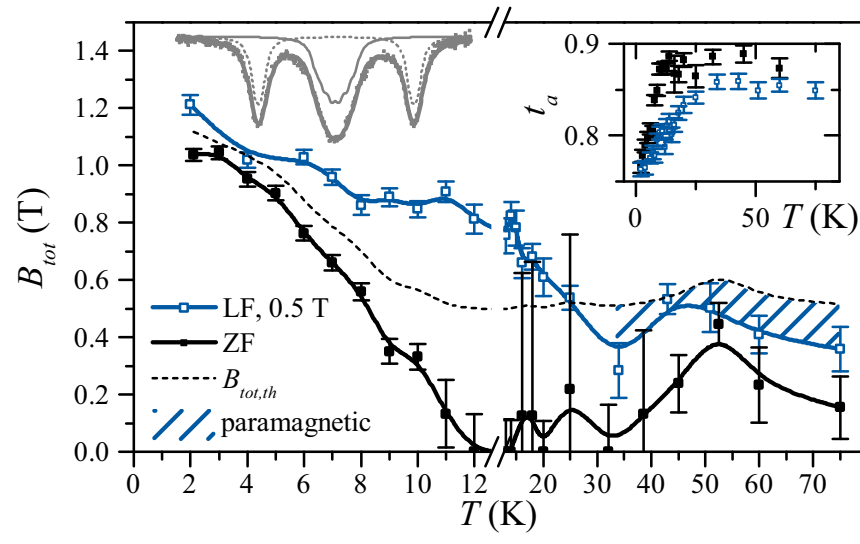

FIG. 2. Results of Mössbauer spectroscopy on the CuFeAs $\mu \mathrm{SR}$ sample. The left inset shows the two component model at $2 \mathrm{~K}$ with $\mathrm{CuFeAs}$ (solid) and ferrocene (dashed, providing experimental linewidth). The increase of magnetic field $B_{\text {tot }}$ below $11 \mathrm{~K}$ is accompanied by a drop of spectral area (right inset), indicating that $\approx 10 \%$ of the Fe nuclei sense much higher fields than quantified by $B_{\text {tot }}$. The transition temperature shifts upon the application of a $0.5 \mathrm{~T}$ longitudinal field (LF) revealing the ferromagnetic character of the magnetic order. Only at the lowest temperatures does a static superposition of zero field (ZF) $B_{\text {hyp }}$ and applied field $B_{e x}$ as described by $B_{\text {tot,theo }}[$ Eq. (1)] apply. In that case dominant AFM order is present.

\section{B. Zero field (ZF) Mössbauer spectroscopy of CuFeAs}

The CuFeAs Mössbauer spectrum mainly consists of a quadrupole doublet (Fig. 2 inset, Fig. 3) with a quadrupole splitting (Table II) typical for 122 compounds [40]. Below $11 \mathrm{~K}$ it broadens by $\approx 0.1 \mathrm{~mm} / \mathrm{s}$. The right side appears broader, which could be associated with a FeAs doublet. However, the absence of this phase in XRD and neutron diffraction and the remaining asymmetry for $15 \mathrm{~K}<T<60 \mathrm{~K}$, i.e., at temperatures where magnetic splitting of the FeAs was expected, suggest a different origin.

The main doublet was modeled in terms of the fully static Hamiltonian assuming an axially symmetric electric field gradient (EFG). Already at room temperature the experimentally observed linewidth $\omega_{s m p}$ (Table II) considerably exceeds the usual spectrometer limit of $0.07 \mathrm{~mm} / \mathrm{s}$, indicating strong distortions of the electronic iron environment especially for the $\mu$ SR sample. This broadening was modeled by a Gaussian

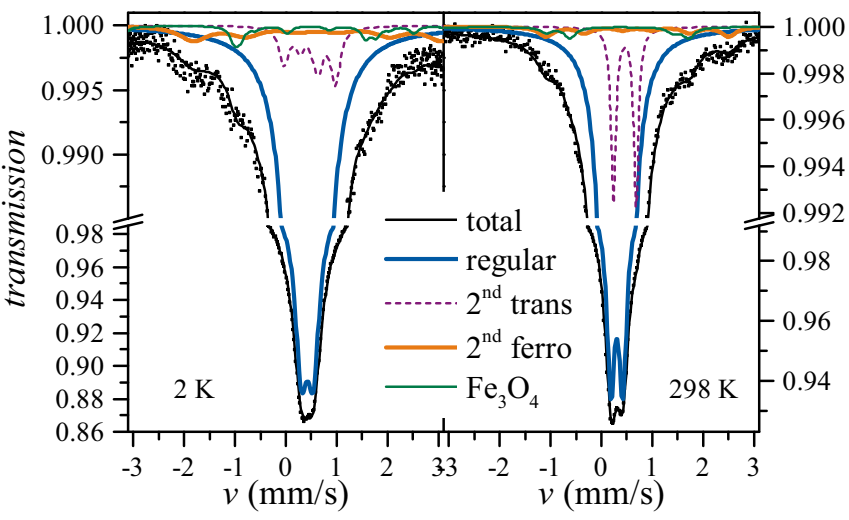

FIG. 3. High statistics Mössbauer spectra of the neutron sample, fit with a four fraction model (parameters in Table III). 
TABLE II. Mössbauer hyperfine parameters of CuFeAs in the single component fit model. $\omega_{s m p}$ refers to the HWHM of lines of the sample spectrum before application of transmission integral. The isomer shift $\delta$ refers to room temperature iron.

\begin{tabular}{lccccc}
\hline \hline Sample & $\begin{array}{c}V_{z z} \\
\left(\mathrm{~V} / \AA^{2}\right)\end{array}$ & $\begin{array}{c}\omega_{s m p} \\
(\mathrm{~mm} / \mathrm{s})\end{array}$ & $\begin{array}{c}\delta(0 \mathrm{~K}) \\
(\mathrm{mm} / \mathrm{s})\end{array}$ & $\begin{array}{c}B_{\mathrm{hyp}} \\
(\mathrm{T})\end{array}$ & $\begin{array}{c}\Delta t_{a} \\
(20 \mathrm{~K} \rightarrow 2 \mathrm{~K})\end{array}$ \\
\hline neutron & $13.2(2)$ & $0.125(3)$ & $0.529(1)$ & $1.06(5)$ & $\begin{array}{c}13(1) \% \\
8(1) \%\end{array}$ \\
\hline \hline
\end{tabular}

distribution of the principal component $V_{z z}$ of the EFG instead of a Lorentzian line broadening. The standard deviations $\sigma_{V z z}=8 \mathrm{~V} / \AA^{2}$ and $4 \mathrm{~V} / \AA^{2}$ for the $\mu \mathrm{SR}$ and neutron sample, respectively, were determined from a global fit of spectra with $T>50 \mathrm{~K}$ and kept constant for the main fit.

The observed magnetic broadening below $11 \mathrm{~K}$ strongly interferes with the experimental variation of the resonance linewidth. The experimental linewidth depends sensitively on the current needle valve passage and evaporation rates of liquid nitrogen and helium. Therefore we attached an additional $12 \mathrm{mg}$ ferrocene absorber to the CuFeAs sample to provide a standard linewidth (see spectrum in Fig. 2). Any additional broadening on top of the standard linewidth and $V_{z z}$ distribution is modeled by $B_{\text {hyp }}>0 \mathrm{~T}$. Using the ferrocene absorber significantly reduces the scattering of the determined total field $B_{\text {tot }}$ (Fig. 2).

For both samples the broadening starts at $\approx 11 \mathrm{~K}$ and almost linearly increases down to $5 \mathrm{~K}$, below which the curve slightly flattens. The broadening corresponds to a hyperfine field $B_{\text {hyp }} \approx 1 \mathrm{~T}$. Assuming an iron pnictide specific conversion ratio of $6.7 \mathrm{~T} / \mu_{\mathrm{B}}$ [40], this corresponds to a magnetic moment of $0.15 \mu_{\mathrm{B}}$. This value is somewhat lower than the saturation moment between 0.2 and $0.3 \mu_{B} / \mathrm{Fe}$, which the magnetization measurements suggest [26,27]. In the same temperature interval the total modeled absorption, measured by the effective thickness $t_{a}$ (Fig. 2, inset), decreases by $\approx 10 \%$, indicating that parts of the previous doublet now sense hyperfine fields much stronger than $1 \mathrm{~T}$, so that they are not captured by the single site model anymore.

We applied a $0.5 \mathrm{~T}$ field parallel to the gamma beam to study the response of the measured magnetic field at the nucleus site. With respect to such a small external field a typical AFM ground state in iron pnictides is expected to be rigid [41], so that a simple superposition of intrinsic $B_{\text {hyp }}$ and applied field $B_{e x}$ should be observed. This leads to the following expression if powder averaging is applied:

$$
\begin{aligned}
B_{\mathrm{tot}} & =\frac{1}{2} \int_{0}^{\pi} d \theta \sin \theta \sqrt{B_{e x}^{2}+B_{\mathrm{hyp}}^{2}-2 \cos ^{2} \theta B_{\mathrm{hyp}} B_{e x}} \\
& =\frac{\left|B_{e x}+B_{\mathrm{hyp}}\right|^{3}-\left|B_{e x}-B_{\mathrm{hyp}}\right|^{3}}{6 B_{e x} B_{\mathrm{hyp}}} .
\end{aligned}
$$

For the analysis we applied the same model as for the $\mathrm{ZF}$ data except that we took $0.5 \mathrm{~T}$ magnetic splitting of the ferrocene doublet into account. In Fig. 2 the result of this longitudinal field (LF) investigation is shown. The onset of broadening shifts to $\approx 25 \mathrm{~K}$ and the curve is not reproduced by Eq. (1) even though for the lowest temperatures the experimental data and theoretical superposition approach each other. In the same way the absorption loss measured by means of $t_{a}$ is shifted to higher temperatures and does not reach the zero field values even above $25 \mathrm{~K}$. This strongly suggests a field induced ferromagnetism and a dominant ferromagnetic character of the observed magnetic order.

We took high statistics spectra (Fig. 3) to both clarify the absorption loss in the magnetic phase and quantify the secondary doublet causing the asymmetry of the main doublet. A $10 \% \mathrm{Fe}_{3} \mathrm{O}_{4}$ fraction $f_{\mathrm{Fe} 3 \mathrm{O} 4}$ was modeled according to the data of Dézsi et al. [42], consistent with neutron diffraction. In addition to the main doublet with larger linewidth at low temperatures, two further subspectra were introduced: a doublet " $2^{\text {nd }}$ trans" responsible for the main doublet's asymmetry at high temperatures and a sextet (" 2 nd ferro") modeling the additional magnetic component underneath the main signal. For the " $2^{\text {nd }}$ trans" component we chose an angle of $90^{\circ}$ between the EFG- $z$ axis and the small transferred magnetic hyperfine field at $2 \mathrm{~K}$ to achieve a reasonable temperature dependence of the center shift. The corresponding fit are presented in Fig. 3 and in Table III. The data support the picture of spectral weight being transferred from the paramagnetic main doublet to a broad magnetic component underneath. The hyperfine parameters of the secondary phases deserve special attention: The $V_{z z \text {,para }}$ and $B_{\text {hyp,ferro }}$ values are comparable to hyperfine parameters of the second $\mathrm{Fe}$ site in $\mathrm{Fe}_{2} \mathrm{As}$ [43,44], which corresponds to the $\mathrm{Cu}$ site in $\mathrm{CuFeAs}$. The sum of fractions $f_{2 n d \text { trans }}+f_{2 n d \text { ferro }}=0.09$ seems temperature independent. Considering $\mathrm{Fe}_{2} \mathrm{As}$ was used as a precursor in the synthesis it seems possible that there is a finite occupancy of the $\mathrm{Cu}$ site in $\mathrm{CuFeAs}$ by $\mathrm{Fe}$. In that case locally a $\mathrm{Fe}_{2}$ As structure appears.

TABLE III. Hyperfine parameters of the high statistics Mössbauer spectra of the neutron sample, shown in Fig. 3.

\begin{tabular}{lccccc}
\hline \hline Subspec. & $T(\mathrm{~K})$ & $V_{z z}\left(\mathrm{~V} / \AA^{2}\right)$ & $\delta(\mathrm{mm} / \mathrm{s})$ & $B_{\text {hyp }}(\mathrm{T})$ & $f$ \\
\hline regular & 2 & $14.1(1)$ & $0.531(1)$ & $0.6(1)$ & $0.81(2)$ \\
& 298 & $14.07(4)$ & $0.412(1)$ & $2.6(1)$ & $0.82(1)$ \\
$2^{\text {nd }}$ trans & 2 & $26.9(3)$ & $0.66(1)$ & $0.041(7)$ \\
& 298 & $26.9(3)$ & $0.569(2)$ & $15.2(3)$ & $0.064(2)$ \\
$2^{\text {nd }}$ ferro & 2 & & $0.73(4)$ & $11.2(2)$ & $0.047(6)$ \\
& 298 & & const. 5 site model [42] & & $0.022(2)$ \\
$\mathrm{Fe}_{3} \mathrm{O}_{4}$ & 2 & & const. 2 site model [42] & & $0.10(1)$ \\
& 298 & &
\end{tabular}




\section{Transverse field (TF) Mössbauer spectroscopy of CuFeAs}

We applied an external field $B_{e x} \leqslant 6.3 \mathrm{~T}$ to study locally the susceptibility of the magnetic moments to the field at $2 \mathrm{~K}$. The field was applied transverse to the gamma beam to allow the Mössbauer source to reside in an almost field free position close to the sample. The ${ }^{57} \mathrm{Fe}$ probe measures the vector sum of the internal $\mathbf{B}_{\text {hyp }}$ and the applied field $\mathbf{B}_{\mathbf{e x}}$. When the moments get aligned to the external field the applied field will be reduced by the internal field due to the negative sign of the Fermi contact interaction. On the other hand moments will align antiferromagnetically to each other due to the exchange field $B_{J}$ and will be pinned to an anisotropy axis described by anisotropy field $B_{A}$. Large $B_{J}$ and/or $B_{A}$ prohibit a polarization, leading to a line broadening as a result of the random superposition of $\mathbf{B}_{\text {hyp }}$ and $\mathbf{B}_{\mathbf{e x}}$. We applied the uniaxial antiferromagnetic Hamiltonian. Its solution describes the energy dependence of the polar angles $\theta_{i}$ of the two moments and $\xi$ of the anisotropy axis with respect to the external field.

$$
\begin{aligned}
E \propto & -B_{J} \cos \left(\theta_{1}-\theta_{2}\right) \\
& -\frac{B_{A}}{2}\left(\cos ^{2}\left(\theta_{1}-\xi\right)+\cos ^{2}\left(\theta_{2}-\xi\right)\right) \\
& -B_{e x}\left(\cos \left(\theta_{1}+\theta_{2}\right)\right)
\end{aligned}
$$

The bipod of EFG $z$ axis and $\mathbf{B}_{\mathbf{A}}$ is randomly distributed with respect to the fixed laboratory frame, i.e., to the gamma ray and $\mathbf{B}_{\mathbf{e x}}$, which are perpendicular to each other. We chose 35 equally distributed orientations of the anisotropy field $\mathbf{B}_{\mathbf{A}}$, each allowing for five orientations of the EFG- $z$ axis perpendicular to $\mathbf{B}_{\mathbf{A}}$ consistent with ordinary stripe AFM order. Although the total number of 175 subspectra seems rather low to simulate the AFM powder in a transverse field, we found no significant changes in a simulation with a higher number of sampling orientations.

The neutron sample was measured in $B_{e x}=6.3 \mathrm{~T}$ at $2 \mathrm{~K}$ (Fig. 4). Three fractions are necessary to reproduce the data: $41(4) \%$ paramagnetic, $44(5) \%$ antiferromagnetic $\left[B_{A}=1.5 \mathrm{~T}, B_{J}=28(10) \mathrm{T}, B_{\text {hyp }}=2.2(2) \mathrm{T}\right]$, and $15(1) \%$ ferromagnetic $\left(B_{A}=B_{J}=0 \mathrm{~T}, B_{\mathrm{hyp}}=9.2 \mathrm{~T}\right)$. This analysis assumed a fixed anisotropy field $B_{A}=1.5 \mathrm{~T}[40,41]$. In the $\mu$ SR sample the corresponding fractions are $40(2) \%, 40(2) \%$,

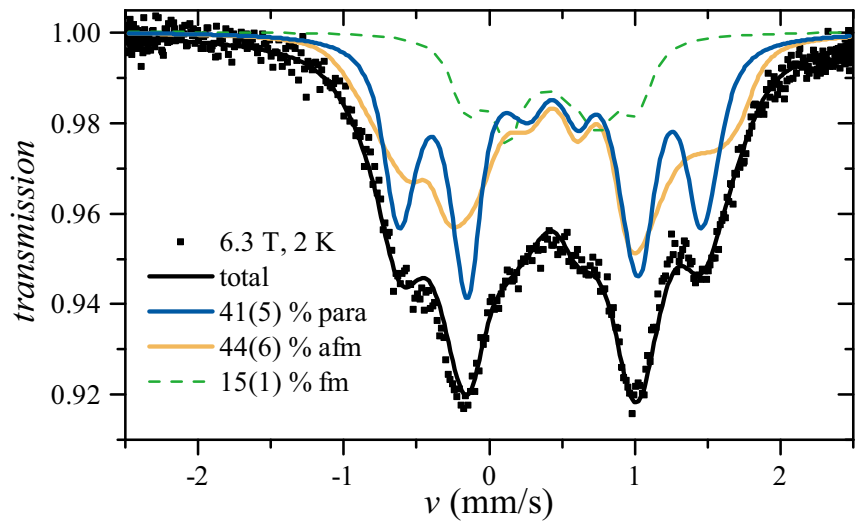

FIG. 4. CuFeAs Mössbauer spectrum of the neutron sample at $2 \mathrm{~K}$ in $6.3 \mathrm{~T}$ applied transverse field. and 20(1)\%, but the corresponding fields can not be determined independently.

These ferromagnetic contributions in this experiment can be identified with the secondary iron in ZF. Due to the large applied field the fraction of these iron atoms with a large on-site magnetic moment appears increased as a result of induced ferromagnetism. The regular iron site, which showed a net magnetic hyperfine field of $\approx 1 \mathrm{~T}$ in $\mathrm{ZF}$, obviously required a more differentiated description in TF. It seems that half of the iron sites behave paramagnetically, whereas the other half exhibits $\approx 0.3 \mu_{\mathrm{B}}$ on-site magnetic moment. We note that this picture presents the easiest model consistent with our data. Eventually the necessity of two components for the regular iron site indicates a magnetic disorder in the main $\mathrm{Fe}$ layer.

\section{D. $\mu \mathrm{SR}$ on CuFeAs}

The $\mu$ SR investigations focus on temperatures $T \leqslant 32 \mathrm{~K}$. The zero field $(\mathrm{ZF})$ muon spin polarization is exponentially damped at $32 \mathrm{~K}$ (Fig. 5), which is probably caused by diluted magnetic impurities. However, at lower temperatures the polarization approaches zero evidencing an additional dynamic relaxation. Below $15 \mathrm{~K}$ an increasing fraction of the muons relax very fast $\left(>5 \mu \mathrm{s}^{-1}\right)$, indicating static magnetic order.

A two component model is sufficient to describe the data:

$$
\begin{aligned}
\frac{A(t)}{A_{0 j}}= & f_{\mathrm{mag}} \cos \varphi_{j}\left(2 / 3 \cdot e^{-\lambda_{T} t}+1 / 3 \cdot e^{-\left(\lambda_{\mathrm{env}} t\right)^{\beta}}\right) \\
& +\left(1-f_{\mathrm{mag}}\right) \cos \varphi_{j} G_{L K T}\left(t, \lambda_{0}\right) e^{-\left(\lambda_{\mathrm{env}} t\right)^{\beta}}
\end{aligned}
$$

The index $j$ indicates the detector pair, either up-down (ud) or forward-backward (fb). $\varphi_{j}$ quantifies the initial rotation of the muon and couples the data of both detector pairs by $\varphi_{f b}-\varphi_{u d}=90^{\circ}$ due to the experimental arrangement of the detectors. The model consists of a magnetically ordered volume fraction $f_{\text {mag }}$ and a paramagnetic fraction $\left(1-f_{\mathrm{mag}}\right)$. The latter is described by the Lorentz-Kubo-Toyabe function $G_{L K T}$. The static damping rate $\lambda_{0}=0.17(1)$ at $32 \mathrm{~K}$ due to magnetic impurities was fixed for all temperatures.

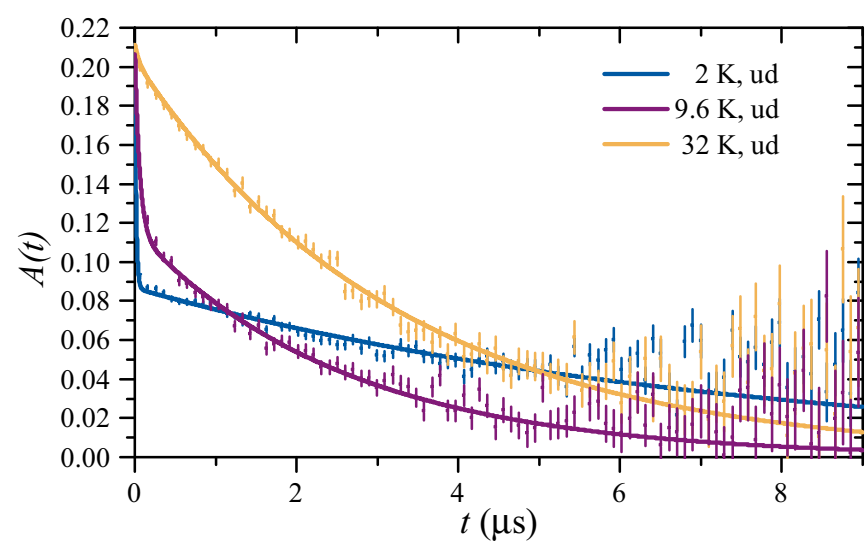

FIG. 5. Asymmetry of the muon spin polarization at zero field. The fast relaxation for low temperatures indicates static magnetic order. 


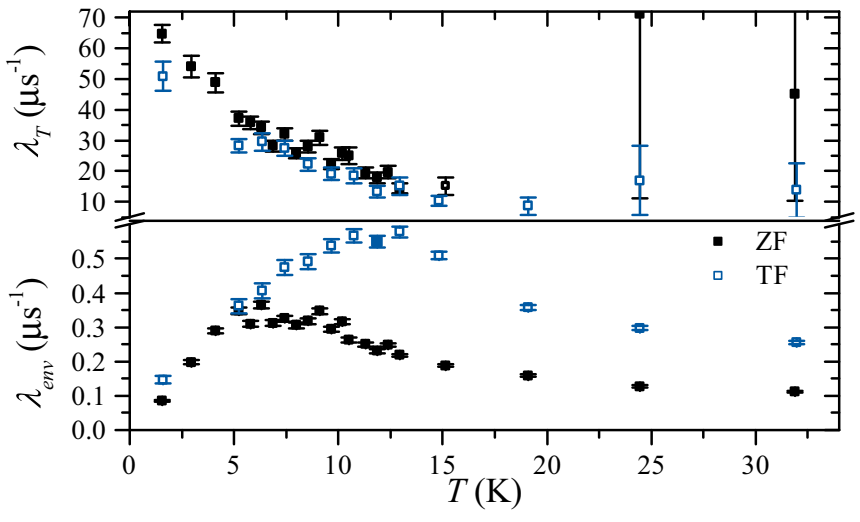

FIG. 6. Relaxation rates of the muon spin polarization functions.

In typical magnetically ordered powder samples $1 / 3$ of the muon spin polarization (called the "tail") is sustained or relaxes with the rate $\lambda_{\text {env }}$. Due to experimental reasons the tail fraction is slightly decreased in the $\mathrm{fb}$ and increased in the ud direction. We assume that the muon thermalizes to an elevated position between four iron moments, similar to other iron arsenides [45-47]. In $\mathrm{NaFe} A s$ the absolute value of the largest precession frequency is consistent with such a muon position and a moment of $\approx 0.3 \mu_{B}$, which is observed experimentally $[48,49]$, so there is good reason to assume the usual muon position in the 111 compounds albeit theoretical calculations are missing so far.

The stretching exponent $\beta$ turns out to be 1 for $T>12 \mathrm{~K}$ and $\langle\beta\rangle=0.79$ for $T<10 \mathrm{~K}$. The peak of the dynamic relaxation rate $\lambda_{\text {env }}$ below $10 \mathrm{~K}$ (Fig. 6) monitors the slowing down of electronic spin fluctuations close to the magnetic phase transition. This shows that the relaxation is dominated by the iron magnetism at low temperatures. The theory for magnetically disordered crystals predicts $\beta=0.5$ in the fast fluctuation limit [50]. It can be concluded that the iron magnetic order has either diluted defects or consists of large diluted moments, or both. On the other hand at higher temperatures the fluctuation rates of iron moments are too fast for the time window of $\mu \mathrm{SR}$. In that case relaxation is mainly due to more homogeneously disordered nuclear magnetic moments justifying $\beta=1$.

The magnetic volume fraction $f_{\text {mag }}$ continuously increases below $20 \mathrm{~K}$. A small fraction 10(1)\% remains "nonmagnetic" corresponding to diamagnetic $\mathrm{Cu}_{3} \mathrm{As}$. A constant $6 \%$ magnetically ordered fraction may be attributed to regions with substantial $\mathrm{Fe}$ occupancy of the $\mathrm{Cu}$ sites, called $\mathrm{Fe}_{2}$ As-like in Fig. 7. A fit of the temperature dependence of the remaining $f_{\text {mag }}$ with Gaussian error function yields $T_{c}=11.2(5) \mathrm{K}$ taking a $50 \%$ criterion as a basis. All these results are in accordance with XRD and susceptibility measurements [26].

The transverse field measurements were carried out to verify the volume fractions extracted from ZF. The $1.6 \mathrm{~K}$ TF spectra are shown in Fig. 8. The data were fit using the following model.

$$
\begin{aligned}
\frac{A(t)}{A_{0 j}}= & f_{\text {mag }} \cos \varphi_{j}\left(\left(1-f_{\text {tail }}\right) \cdot e^{-\lambda_{T} t}+f_{\text {tail }} \cdot e^{-\lambda_{\text {env }} t}\right) \\
& \left.+\left(1-f_{\text {mag }}\right) \cos \left(\gamma B_{W T F} t+\varphi_{j}\right)\right) e^{-\lambda_{\text {env }} t}
\end{aligned}
$$

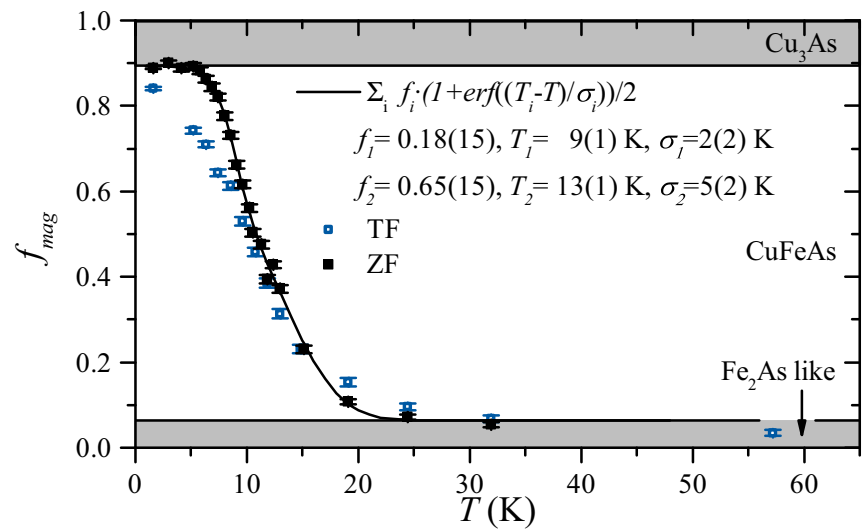

FIG. 7. Magnetic volume fraction in $\mathrm{CuFeAs}$ derived from $\mathrm{ZF}$ and $\mathrm{TF} \mu \mathrm{SR}$ experiments. The solid line is a phenomenological fit using two error functions (erf) quantifying the magnetic inhomogeneity. The discrepancy between ZF and TF data probably shows that for $T<10 \mathrm{~K}$ internal fields are partly smaller than 5 $\mathrm{mT}$. On the other hand the applied field induces ferromagnetism at $\approx 20 \mathrm{~K}$.

Inherently, the TF data give a lower limit for the magnetic volume fraction and partly fall $10 \%$ below the $\mathrm{ZF}$ data. This may be caused by the fraction of small fields $B \leqslant B_{e x}$, which contribute to the ZF magnetic fraction but will cause a coherent precession in a small field and thus contribute to the paramagnetic fraction in TF [51]. Since the model only contains a single nonmagnetic signal fraction describing both paramagnetic $\mathrm{CuFeAs}$ and diamagnetic [52] $\mathrm{Cu}_{3} \mathrm{As}$ the muon spin precession frequency $\omega=\gamma B_{W T F}$ depends on the contributing volume fractions. A nonrelaxing tail in the TF measurements at the lowest temperatures is evident in Fig. 8. Its temperature dependence, shown in the inset, indicates a continuous reduction with increasing temperature due to decreasing magnetic order parameter, i.e., the intrinsic local field at some muon sights becomes as small as the applied field [51]. The presence of the tail unambiguously proves the static character of the magnetic order, however the small $f_{\text {tail }}$

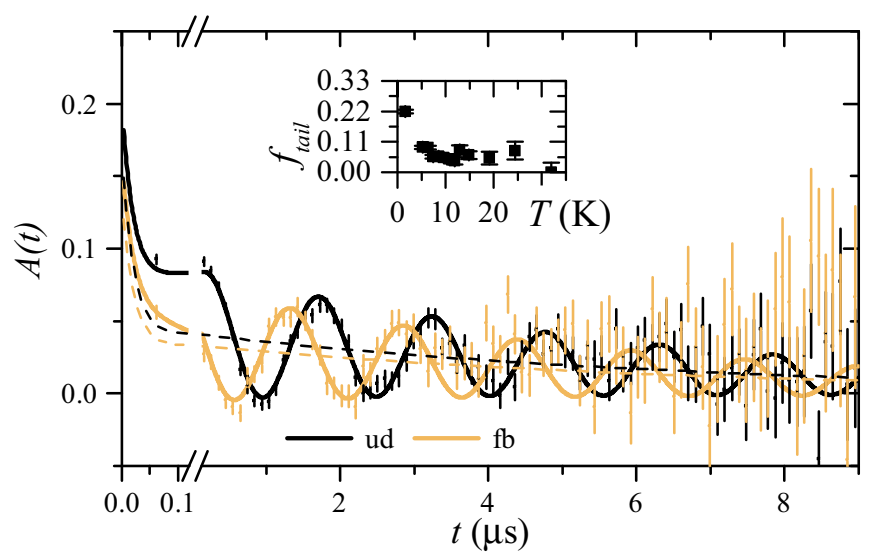

FIG. 8. Asymmetry of the muon spin polarization for a transverse field. The oscillating signal is due to paramagnetic and weak magnetically ordered volume fractions, whereas the dashed component with partial tail $\left(f_{\text {tail }}\right.$, inset $)$ corresponds to static magnetic order. 
indicates that these static magnetic fields differ strongly in magnitude.

\section{E. Search for magnetic order using neutron diffraction on CuFeAs}

High intensity diffraction data were measured at $2.5 \mathrm{~K}$ and $20 \mathrm{~K}$ to search for magnetic Bragg peaks indicative of long range magnetic order [Fig. 9(a)]. By subtracting the $20 \mathrm{~K}$ data from the data at lower temperature $(2.5 \mathrm{~K})$, the nuclear Bragg peaks are eliminated (assuming there is no substantial magnetic coupling to the lattice), leaving only the magnetic diffraction pattern [Fig. 9(b)]. No magnetic peaks were found in the subtraction, and hence no long range magnetic order was found. If we assume a propagation wave vector $\boldsymbol{K}=$ $(0,0,0)$, the irreducible representations for the Fe site can be written as $\Gamma_{\text {mag }}=\Gamma_{13}+\Gamma_{16}+\Gamma_{29}+\Gamma_{210}$, where $\Gamma_{13}, \Gamma_{29}$ are ferromagnetic and $\Gamma_{16}, \Gamma_{210}$ are antiferromagnetic. In addition, $\Gamma_{13}$ only allows the spins to point along the $c$ axis and $\Gamma_{29}$ only allows the spins to lie in the $a-b$ plane. The difference data can be used to estimate limits for the Fe ions ordered magnetic moment as $m_{c}<0.1 \mu_{B}$ along the $c$ axis and $m<0.05 \mu_{B}$ in the $a-b$ plane. If we consider antiferromagnetic structures where the magnetic peaks are separated from the structural peaks, the statistical limit on a moment is $m \leqslant 0.04 \mu_{B}$. These limits apply to long range ordered (resolution-limited) magnetic Bragg peaks. The Mössbauer and muon data, on the other hand, suggest that the order is short range in nature. This would broaden any magnetic peaks, making detection of a magnetic signal more difficult and consequently the limit to any possible ordered moment larger.
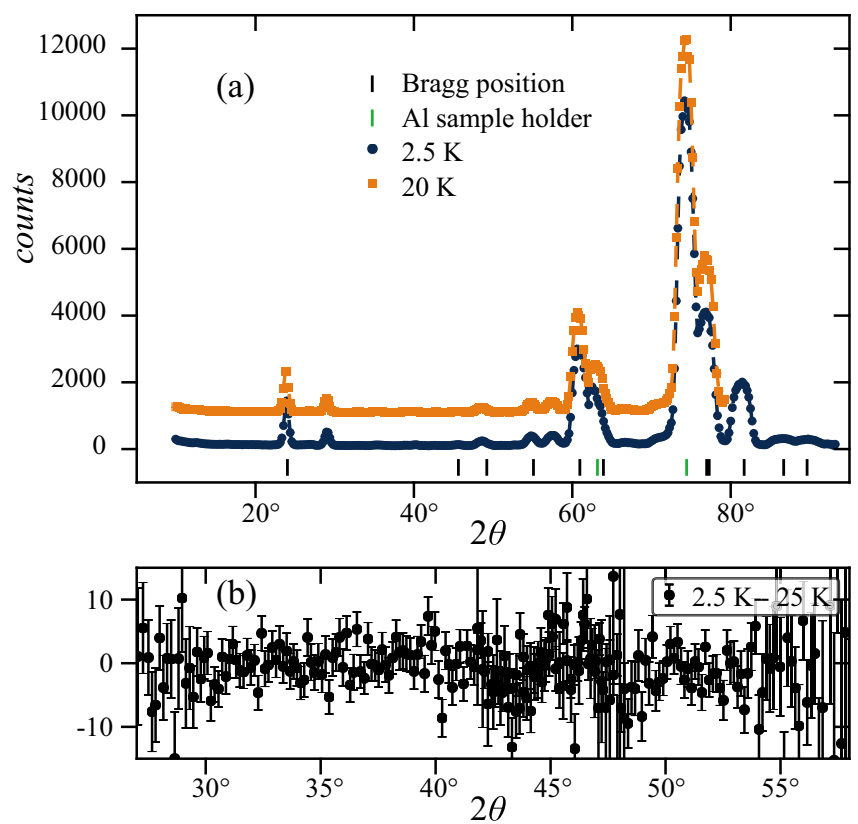

FIG. 9. Neutron powder diffraction pattern of CuFeAs collected at 2.5 and $20 \mathrm{~K}$ (orange squares and blue circles, respectively) $(\lambda=$ $2.44 \AA$ ). Bottom plot indicates the difference in diffraction intensities collected at $2.5 \mathrm{~K}$ and $20 \mathrm{~K}$. Peaks at $2 \theta \approx 63.2^{\circ}$ and $\approx 74.4^{\circ}$ are from the $\mathrm{Al}$ sample holder.

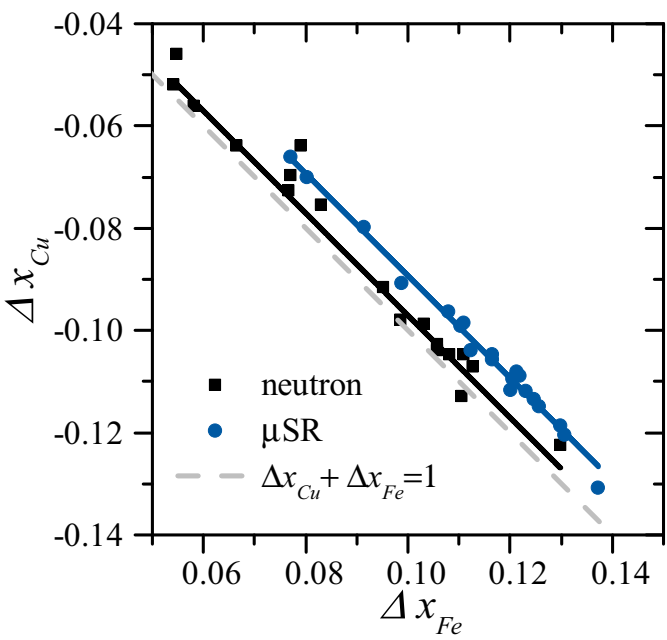

FIG. 10. $\mathrm{Cu}_{1+\Delta x_{C u}} \mathrm{Fe}_{1+\Delta x_{F e}}$ As elemental deviations for $\mu$ SR and neutron samples, probed by WDX. It suggests that the $\mathrm{Cu}$ sites have some limited occupation by Fe.

\section{F. Local elemental constitution of $\mathrm{CuFeAs}$ probed by WDX}

With the findings of secondary $\mathrm{Fe}$ sites and the discrepancy of $\mathrm{Cu}-z$ positions between XRD [26] and neutron diffraction, which can interfere with the site occupancy, we reinvestigated both samples by means of wavelength dispersive $\mathrm{X}$-ray spectroscopy (WDX). The averaged composition is $\mathrm{Cu}_{0.91(2)} \mathrm{Fe}_{1.13(2)} \mathrm{As}$ for the $\mu$ SR sample and $\mathrm{Cu}_{0.91(3)} \mathrm{Fe}_{1.10(2)} \mathrm{As}$ for the neutron sample. Because both XRD and neutron diffraction state a full occupancy of the crystallographic site we act on the assumption of a considerable occupancy of the $\mathrm{Cu}$ site by iron. The anticorrelation of $\mathrm{Cu}$ and Fe site occupancy takes place not only in average, but on every single data point of both samples. In Fig. 10 we show the data of the individual data points. The solid lines correspond to a linear fit with fixed slope -1 , obviously describing the data. The large standard variations for both element $\mathrm{Fe}$ and $\mathrm{Cu}$ compared to the $\mathrm{As}$ value reflect the significant variability of the composition of the phase for $\mathrm{Fe}$ and $\mathrm{Cu}$. The increased excess iron content of the $\mu \mathrm{SR}$ sample is responsible for the shift of the corresponding data in Fig. 10. The increased structural disorder may be responsible for the increased paramagnetic linewidth of the $\mu$ SR sample in Mössbauer spectroscopy.

\section{G. Theoretical investigation}

For different samples, various $\mathrm{Cu}$ content and atomic positions have been reported [26,27]. However, FeAs-based compounds are well known for their strong sensitivity of the magnetism to the internal coordinates, in particular to the As position $z_{A s}[37,53]$. We focus our theoretical investigation to construct a relevant model, which describes the influence of (i) the $\mathrm{Cu}$ content and (ii) the As position $z_{A s}$ on the electronic and magnetic properties.

To gain deeper insight into the electronic structure and the orbital occupations of $\mathrm{CuFeAs}$ we calculate the density of states (DOS). Figure 11 shows the nonmagnetic total and site-resolved calculated DOS for the fully occupied copper site. In accordance with similar FeAs-based compounds, Fe-3d 


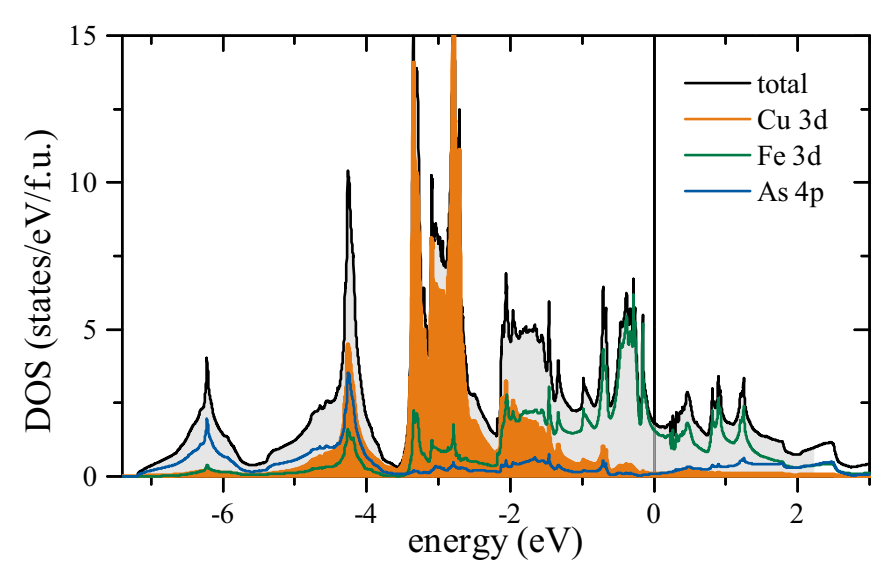

FIG. 11. Calculated nonmagnetic total and site-resolved density of states for stoichiometric CuFeAs. The states close to the Fermi energy $\left(E_{F}\right)$ consist mainly of $\mathrm{Fe}-3 d$ contributions. The $\mathrm{Cu}-3 d$ states are essentially fully occupied. The contribution of the copper $3 d$ states to the Fermi surface is very small.

states dominate clearly in the vicinity of the Fermi level, in contrast the $\mathrm{Cu}-3 d$ bands lie in a region of deeper energy centered at about $-3 \mathrm{eV}$ and exhibit certain analogy to metallic $\mathrm{Cu}$ (not shown here). The $\mathrm{Cu}$ contribution at the Fermi level is rather small, comparable with the contribution of As.

Regarding the low lying, essentially fully filled $\mathrm{Cu}-3 d$ states, we assume that each $\mathrm{Cu}$ transfers one valence electron $(4 s)$ to the valence band. Thus, one electron was chosen for the VCA treatment. For the largest $\mathrm{Cu}$ deficiency, a $\mathrm{Cu}$ occupancy of 0.789 was noted by Qian et al. [27]. Thus, we simulated a $\mathrm{Cu}$ content in a range of $0.8-1.2$ within the VCA.

VCA was demonstrated to describe successfully electronic and magnetic properties in good approximation in Fe pnictides on different sites upon doping [37,54,55]. Due to the layered nature of the systems, it works particularly well for the anion site: If the hybridization of a specific site with the relevant magnetic states is small (as in our case), VCA will reflect the changes with respect to band filling and the change in the averaged crystal potential rather well. In the case of $\mathrm{Cu}_{y} \mathrm{FeAs}, y<1$ describes $\mathrm{Cu}$ vacancies (hole doping) in the $\mathrm{Cu}$ layer, whereas $y>1$ describes the situation of electron doping. The VCA calculations are expected to model the respective changes of the magnetism for the $\mathrm{Fe}$ site of the $\mathrm{Fe}$-As layers; the behavior of $\mathrm{Fe}$ at the $\mathrm{Cu}$ site is not covered by this VCA approach.

Figure 12 visualizes the results of these calculations: For the experimental structure data we find magnetic order for full $\mathrm{Cu}$ occupation (stoichiometric compound). The relaxation of $\mathrm{Cu}$ with respect to the total energy has almost no influence on the structure and only a small influence on the magnetic moment. As known from previous studies of Fe-pnictide compounds, the calculated relaxed As position $z_{A s}=0.2490$ yields short Fe-As bonds and a too small $h_{\text {anion }}=1.455 \AA$ compared to experimental data. However, an underestimate of less than $0.1 \AA$ compared with the neutron data $\left(h_{\text {anion }}=1.53 \AA\right.$, Table $\left.\mathrm{I}\right)$ is well within the usual discrepancy, giving strong support for the latter and strongly disfavoring the much larger value from XRD $\left(h_{\text {anion }}=1.738 \AA\right.$ [26]). The relaxation of both sites, As and $\mathrm{Cu}$, yields essentially the same results, see Fig. 12 .

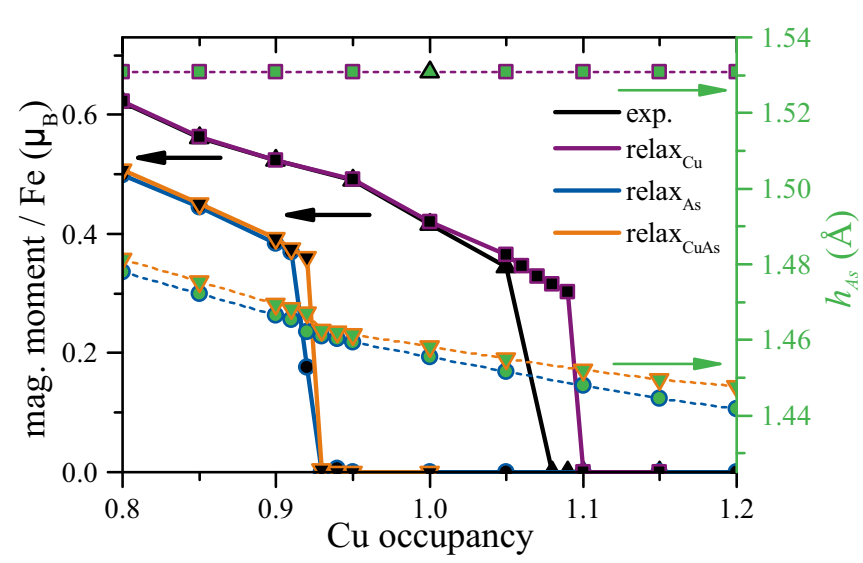

FIG. 12. Dependence of the arsenic height $h_{A s}$ (green) and the magnetic moment (black) on the formal $\mathrm{Cu}$ occupancy $y$ for experimental and relaxed atom positions of $\mathrm{Cu}_{y} \mathrm{FeAs}$. Experimental atomic positions are obtained from neutron powder diffraction data.

We conclude that an accurate As position is crucial for the description of the magnetic moment in $\mathrm{CuFeAs}$, whereas the details of the $\mathrm{Cu}$ position have only a minor influence.

The magnetic moments for unrelaxed (experimental) and relaxed $\mathrm{Cu}$ data points have almost the same values. Due to the larger Fe-As distance they are comparatively large and vary in a range of $0.6-0.35 \mu_{\mathrm{B}} / \mathrm{Fe}$ for a formal Cu occupancy lower than 1.05. At the critical region between $1.05<y<$ 1.1 (electron doping) magnetism collapses and the compound becomes nonmagnetic for $y>1.1$. Almost the same behavior, though shifted by $\approx 13 \%$ with respect to the $\mathrm{Cu}$ occupancy, is obtained for the magnetic moments for relaxed As and relaxed As and $\mathrm{Cu}$ positions. Their critical $\mathrm{Cu}$ occupancy values lie at $y \approx 0.92$ and induce a kink in the $h_{A s}$ position curve. This shows the presence of a sizable coupling of magnetic moments and lattice in this compound.

A closer look onto the related changes of the calculated nonmagnetic (As and $\mathrm{Cu}$ relaxed) DOS reveals sizable differences in the number of Fe-3d states at the Fermi level. Similar to $\mathrm{Ba}_{1-x} \mathrm{~K}_{x}\left(\mathrm{Fe}_{1-y} \mathrm{Co}_{y}\right)_{2} \mathrm{As}_{2}$ [54], there are two different regions: For $y=0.8$ and 0.85 the Fe-3d DOS values lie between 1.94 and 1.90 states $/ \mathrm{eV} /$ f.u. and thereby fulfill the Stoner criterion $\left[N\left(E_{F}\right) \geqslant 1 / I\right.$ with $I \sim 1 \pm 0.1$ for Fe-3d states] [56]. For the second region with $\mathrm{Cu}$ occupancy between $y=0.9$ and stoichiometric $\mathrm{CuFeAs}$ the $\mathrm{Fe}-3 d$ contribution decreases rapidly. The values between 1.64 and 1.43 states/eV/f.u. are far from fulfilling the criterion and therefore the magnetism is disfavored.

In contrast to previous samples, where $\mathrm{Cu}$ deficiency was observed, our samples show a partial substitution of $\mathrm{Cu}$ by $\mathrm{Fe}$. To study the local influence of $\mathrm{Cu}$-Fe-replacement we constructed a supercell containing $25 \% \mathrm{Fe}$ on the $\mathrm{Cu}$ site (lower panel in Fig. 13, purple colored atom in inset). The total density of states of the low lying valence states (not shown) show a rigid bandlike behavior for a large part of the valence states. Especially states with dominating $\mathrm{As}$ and $\mathrm{Cu}$ contribution at the band bottom are rigidly shifted to higher energies by about $130 \mathrm{meV}$. With respect to the VCA, modeling the hole doping for $\mathrm{Cu}$ deficiency, the change in the averaged crystal potential 


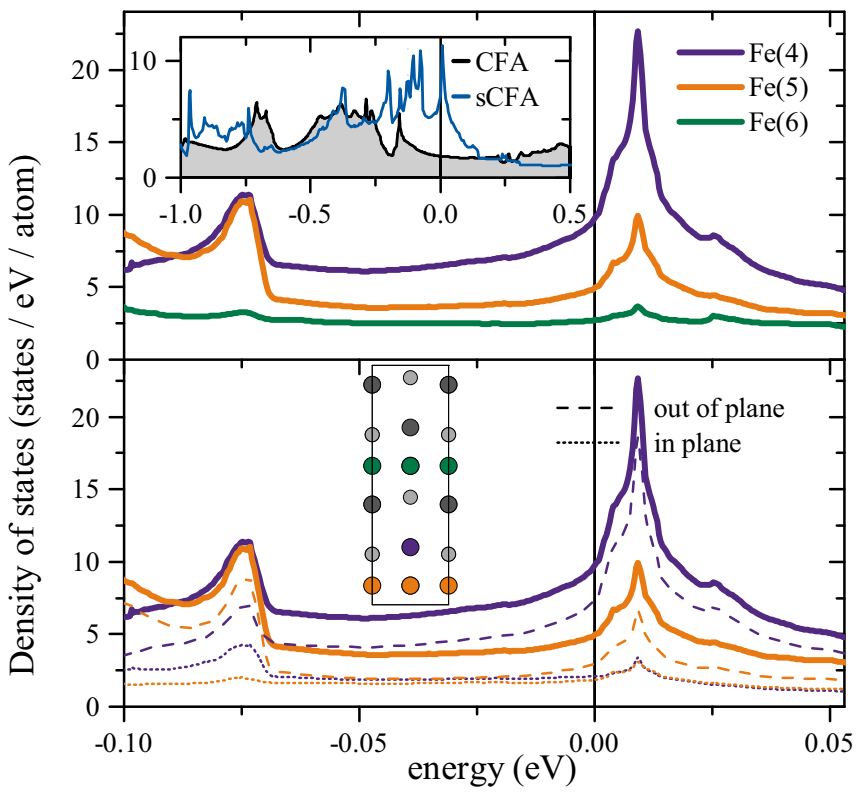

FIG. 13. Upper panel: Calculated atom-resolved density of states for $\mathrm{Cu}_{0.75} \mathrm{Fe}_{1.25} \mathrm{As}$. The additional $\mathrm{Fe}$ atom, replacing $\mathrm{Cu}[\mathrm{Fe}(4)$ on the Wyckoff position $1 \mathrm{~b}$, see inset], yields a strong increase in the density of states near the Fermi level $E_{F}$. The next nearest $\mathrm{Fe}$ atom [Fe(5)] also shows an increased $\operatorname{DOS}\left(E_{F}\right)$ due to sizable hybridization, while the distant $\mathrm{Fe}$ layer $[\mathrm{Fe}(6)]$ gives a small contribution, only. Inset: Comparison of total density of states near Fermi level of stoichiometric $\mathrm{CuFeAs}$ (CFA) and the substituted $\mathrm{CuFeAs}$ (sCFA). The $\operatorname{DOS}\left(E_{F}\right)$ increases from 1.8 to 7.0 states/eV/f.u. Lower panel: Display of the dominant $3 d$ orbitals (in-plane vs out-of-plane) for $\mathrm{Fe}(4)$ and $\mathrm{Fe}(5)$. Inset: Model of the calculated $\mathrm{Fe}$ substituted $\mathrm{Cu}_{0.75} \mathrm{Fe}_{1.25} \mathrm{As}$ (space group $P 4 \mathrm{~mm}$ ), the different Fe sites are colored corresponding to the DOS graphs.

(for $25 \% \mathrm{Fe}$ ) corresponds to a $\mathrm{Cu}$ occupation $\mathrm{y} \sim 0.85$ (comp. Fig. 12).

In contrast to the low lying bands, the states in the vicinity of the Fermi level strongly differ from a rigid band behavior. Here the substituted $\mathrm{Fe}$ [Fe(4), see inset Fig. 13] forms a rather localized state, yielding a DOS peak close to the Fermi energy (see upper panel in Fig. 13 and inset). Fig. 13 also visualizes the strong hybridization of the additional $\mathrm{Fe}(4)$ atom with the nearest $\mathrm{Fe}$ atoms $(\mathrm{Fe}(5)$ showing a similar, though less pronounced peak), while to the more distant $\mathrm{Fe}(6)$ layer shows essentially no hybridization effects.

However, compared to the experimental determined $\mathrm{Fe}$ excess of 10-13\%, our supercell contains about twice the amount of excess Fe [57]. Thus, for the averaged crystal potential (compared to VCA) the realistic Fe excess should correspond to a $\mathrm{Cu}$ content close to the magnetic instability for the original Fe sites (see Fig. 12). Experimentally, no structural order of the excess Fe was observed. For randomly distributed excess $\mathrm{Fe}$, the original $\mathrm{Fe}$ sites have in average only about one neighboring excess Fe atom. This leads to the question how stable the localized $\mathrm{Fe}(4)$ state is with respect to the smaller Fe(4) excess. Therefore, we analyzed the contributing orbitals and found that the localized $\mathrm{Fe}(4)$ state is dominated by its out-of-plane orbitals [similar, but less pronounced for $\mathrm{Fe}(5)]$. One would expect that the dilution of $\mathrm{Fe}(4)$ atoms in the layer will mainly affect the $\mathrm{Fe}(4)$ in-plane states (since they are strongly interacting with the neighboring in-plane $\mathrm{Fe}(4)$ which are partially removed upon dilution). In consequence, the dilution effect on the out-of-plane states should be weak, therefore only slightly change height and position of the $\mathrm{Fe}(4)$ related DOS peak.

To understand the influence of the Fe substitution on the magnetism more qualitatively, we also performed a spinpolarized calculation for the Fe-excess super cell. As expected from the (nonmagnetic) DOS peak close to $E_{F}$, the substituted $\mathrm{Fe}(4)$ generates a rather large local magnetic moment. For $25 \% \mathrm{Fe}$ excess, the $\mathrm{Fe}(4)$ moment reaches a value of about $2.5 \mu_{\mathrm{B}} / \mathrm{Fe}$, while the $\mathrm{Fe}(5)$ and $\mathrm{Fe}(6)$ show a magnetic moment of about 0.8 and $-0.7 \mu_{\mathrm{B}} / \mathrm{Fe}$, respectively. Relaxation of the $\mathrm{Fe}(4)$ atom with respect to the total energy yields essentially the same result for the site moment: $\mathrm{Fe}(4)=2.7 \mu_{\mathrm{B}}$ and $\mathrm{Fe}(5)=0.7 \mu_{\mathrm{B}}$, while $\mathrm{Fe}(6)$ is reduced to $-0.4 \mu_{\mathrm{B}}$. Taking into account the experimentally observed dilution of $\mathrm{Fe}(4)$ atoms [in average one $\mathrm{Fe}(4)$ neighbor], the averaged magnetic moment of $\mathrm{Fe}(5)$ and $\mathrm{Fe}(6)$ should be further reduced.

In conclusion, our super-cell calculations find that the excess $\mathrm{Fe}$ brings the original Fe layers near the magnetic instability due to effective hole doping (Fig. 12). The effect is enhanced by local polarization due to hybridization of the excess $\mathrm{Fe}$ with the neighboring regular $\mathrm{Fe}$ atoms and a rather large magnetic moment of the excess Fe.

\section{H. Zero field Mössbauer spectroscopy on $\mathrm{CuFeSb}$}

The zero field Mössbauer spectra of $\mathrm{CuFeSb}$ consist of three different signals: two signals that arise from magnetically ordered sites and the third from a $\mathrm{Fe}_{x} \mathrm{Sb}$ impurity phase (Fig. 14). The latter can be identified according to its transition temperatures below $T_{N} \lesssim 60 \mathrm{~K}$ (corresponding to $x \gtrsim 1.3$ ) and the estimated magnetic splitting of $\approx 3 \mathrm{~mm} / \mathrm{s}$ at $4.2 \mathrm{~K}$ $[58,59]$. Because of the distribution of $x$ in this foreign phase, there is no well defined sextet observed. This phase was modeled by a broad doublet. It accounts for the increased

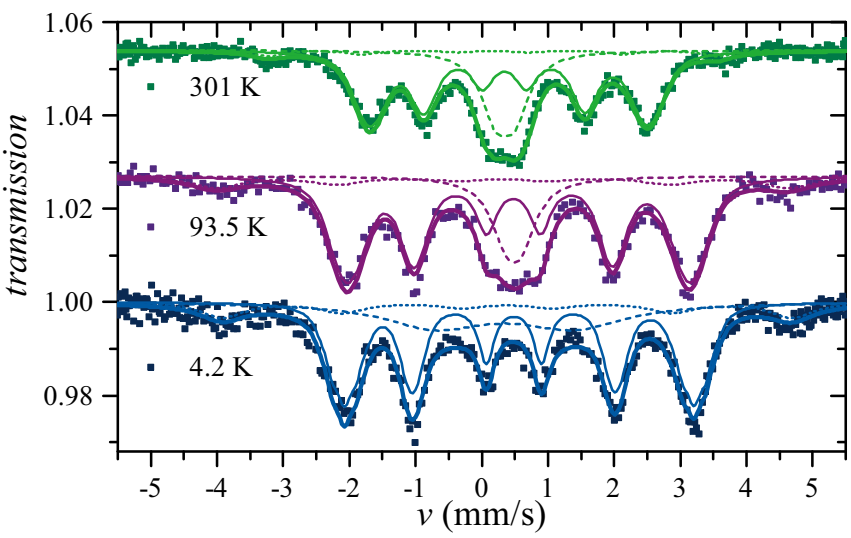

FIG. 14. CuFeSb Mössbauer spectra show magnetic splitting with a saturation field of $16.08(2) \mathrm{T}$. Approximately $10 \%$ of the intensity is assigned to a second sextet identified as a secondary iron at the $\mathrm{Cu}$ position. Spectra at temperatures between $50 \mathrm{~K}$ and $100 \mathrm{~K}$ are dominated by the magnetic phase transition of $\mathrm{Fe}_{\mathrm{x}} \mathrm{Sb}$, which give an impurity signal of $\approx 20 \%$. 


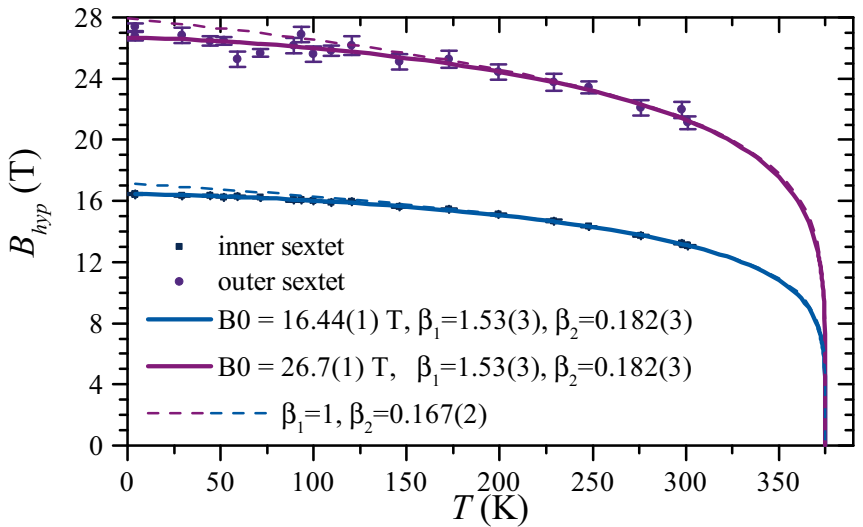

FIG. 15. CuFeSb magnetic order parameter $B_{\text {hyp }}$, modeled by $B(T)=B_{0}\left(1-\left(T / T_{C}\right)^{\beta_{1}}\right)^{\beta_{2}}$, assuming $T_{C}=375 \mathrm{~K}$ [24]. The individual critical exponents do not differ significantly and were set equal. A single critical exponent fit for $T>200 \mathrm{~K}$ (dashed) approaches an exponent of 0.17 .

absorption in the central part of the spectrum. The signal fraction of $\mathrm{Fe}_{x} \mathrm{Sb}$ ranges between $15 \%$ and $20 \%$.

According to Qian et al. [24] in CuFeSb there exists $\mathrm{Cu} / \mathrm{Fe}$-site disorder. We act on the assumption that the outer sextet signal corresponds to an iron site within the $\mathrm{CuSb}$ plane. Thus, the iron moment is enhanced in the Sb neighborhood. Furthermore, the principal component of the EFG, which was assumed axially symmetric for simplicity, is temperature independent and possesses an average value of $-10(1) \mathrm{V} / \AA^{2}$ for the main iron site and an average angle between the principal axis of the EFG and the magnetic moments of $78(6)^{\circ}$. The median angle lies at $89^{\circ}$. The outer sextet was modeled with $V_{z z}=0$, as a further degree of freedom concerning the EFG prohibits a precise determination of the outer magnetic hyperfine field and leads to unphysical isomer shift values. The signal fraction of this outer sextet increases from $7 \%$ to $12 \%$, which is theoretically described by a lower Debye temperature compared to the main site.

As shown in Fig. 15, the magnetic order parameters of the two sextet sites scale by a factor of 1.62(3). With a fixed transition temperature $T_{C}=375 \mathrm{~K}$, a rather low critical exponent $\beta_{\text {crit }}=0.17(1)$ can be assigned to both sextet sites simultaneously. It supports the picture of a $2 \mathrm{D}$ ferromagnetism within the $a b$ plane, as suggested earlier [24]. Qian et al. [24] discussed the significance of the increase of the iron moment below $100 \mathrm{~K}$. Such an increase is not present in the Mössbauer data (Fig. 15). The saturated moment of $\approx 1.6 \mu_{\mathrm{B}}$ observed by Qian et al. leads to a conversion ratio of $10 \mathrm{~T} / \mu_{\mathrm{B}}$ at the regular iron site.

The inner sextet shows a deviation from the expected 3:2:1:1:2:3 pattern for the powder fractions for the following reasons. First the thick absorber preparation leads to a leveling of the line intensities. Secondly there exists a distribution of internal fields according to the composition of the next nearest neighbor environment, which is subject to $\mathrm{Fe}-\mathrm{Cu}$ disorder. This distribution was modeled as Gaussian, using five subspectra representing a Gaussian width of $\sigma_{\text {hyp }}=0.065(3) B_{\text {hyp }}$. As the outer sextet's linewidth will be affected more by the distribution of fields, this results in a decreased height of the

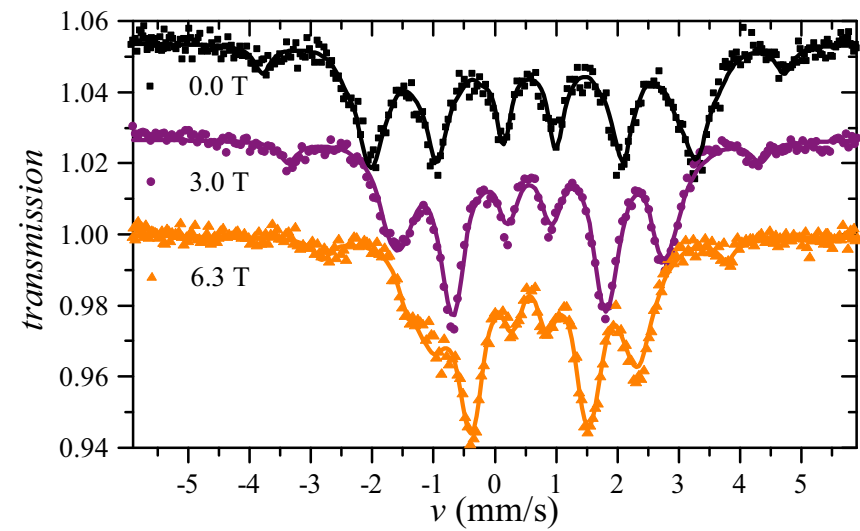

FIG. 16. CuFeSb applied transverse field Mössbauer spectra at $2.1 \mathrm{~K}$, fit by a distribution of internal hyperfine fields (Fig. 17).

outer peaks. Finally, the magnetic splitting of the foreign phase increases the absorption in the central part of the spectrum.

\section{Transverse field Mössbauer spectroscopy of $\mathrm{CuFeSb}$}

$\mathrm{CuFeSb}$ was characterized as a soft ferromagnet [24], i.e., it is easily polarizable at small fields. In Mössbauer spectroscopy this polarization presents itself in two aspects: First the well known 3:2:1:1:2:3 intensity ratio of the sextet lines is valid for a powder sample, but a polarized sample shows a $3: 4: 1: 1: 4: 3$ or 3:0:1:1:0:3 pattern for TF and LF measurements, respectively. Second, as the moments will be well aligned parallel to the field, and not preferentially to an anisotropy axis or plane, there will be no additional field distribution due to the vector addition of internal field $B_{\text {hyp }}$ and external fields $B_{e x}$. Instead, the total field sensed by the iron nuclei is simply the difference of $B_{\text {hyp }}$ and $B_{e x}$, as the Fermi contact interaction has a negative sign.

Our Mössbauer measurements at $2.1 \mathrm{~K}$ confirm these expectations: The total splitting of both sextets decreases simultaneously and linearly by the value of the applied field (Fig. 16), disregarding a constant offset of 0.5(1) $\mathrm{T}$ in both sextets due to the demagnetizing field in powders [60]. In this

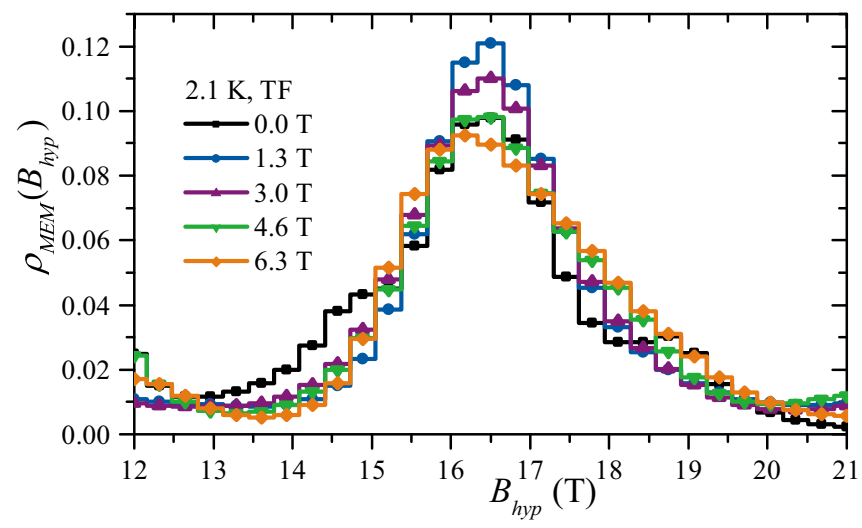

FIG. 17. CuFeSb hyperfine field distribution [extracted using maximum entropy method (MEM)] as a function of applied transverse field (TF). The asymmetry of the distribution of internal fields above $4 \mathrm{~T}$ describes the anomaly in the left peak (negative velocity) of the spectrum (Fig. 16). 
model the impurity again was modeled as a broad peak. It turns out that the width of this peak is minimal at $B_{e x}=3 \mathrm{~T}$. This is in line with the antiferromagnetic character of the $\mathrm{Fe}_{\mathrm{x}} \mathrm{Sb}$ impurity phase, which in principle can be responsible for such an intermediate narrowing.

However, for $B_{e x} \geqslant 4.6 \mathrm{~T}$ there is an anomaly on the left side of the main sextet (Fig. 16). We tried to model this subspectrum by means of $B_{\text {hyp }}$-MEM on top of the randomly distributed EFG within the applied field as described for CuFeAs. In Fig. 17 the resulting distribution $\rho\left(B_{\text {hyp }}\right)$ is shown. According to the $\mathrm{ZF}$ analysis $\rho\left(B_{\text {hyp }}\right)$ is Gaussian at low field but becomes asymmetric at higher fields. This advanced model substantially improves the fit, but due to the absence of anomaly in magnetization measurements [24] this partial increase of the moments of $\approx 10 \%$ in high fields is questionable.

\section{DISCUSSION}

The local probe investigations of our CuFeAs specimens indicate static magnetic order below $11 \mathrm{~K}$ with a short range coherence length, rendering the investigation of the micromagnetic structure impossible by means of neutron diffraction. The gradual increase of both $B_{\text {hyp }}$ (Fig. 2) in Mössbauer spectroscopy and $\lambda_{T}$ (Fig. 6) in $\mu$ SR support the picture of a magnetically inhomogeneous system, even though the complete $\mathrm{CuFeAs}$ phase orders (Fig. 7). Transverse field experiments of both techniques reveal that the size of the magnetic order parameter varies by one order of magnitude.

The application of an external field shifts the onset of line broadening to substantially higher temperatures, suggesting a ferromagnetic character of the magnetism. However, this is inconsistent with high field $(6.3 \mathrm{~T})$ experiments, where the regular iron sites seem to be either antiferromagnetic or paramagnetic, depending on the influence of neighboring secondary iron, but not ferromagnetic.

WDX experiments indicate that $9 \%$ of the $\mathrm{Cu}$ sites are occupied by $\mathrm{Fe}$. A reinvestigation of the neutron diffraction data allows for such a mixing without loss of goodness of fit, if an insignificant total deficiency of $3 \%$ at the $\mathrm{Cu}$ site was assumed. Such a deficiency can occur in LiFeAs [61,62] and was discussed for the case of NaFeAs [63]. The studies of Qian et al. [27] are indeed based on a strongly $\mathrm{Cu}$ deficient $\mathrm{CuFeAs}$. However, the WDX results disfavor a real total deficiency of the $\mathrm{Cu}$ site.

The $9 \%$ secondary $\mathrm{Fe}$ are in line with Mössbauer spectroscopy, where an additional doublet is seen at higher temperature, which than contributes a broad magnetic component below $11 \mathrm{~K}$. The hyperfine parameters of the corresponding Mössbauer subspectra are similar to the secondary site in $\mathrm{Fe}_{2} \mathrm{As}$. In contrast to the antiferromagnetic [44] $\mathrm{Fe}_{2} \mathrm{As}$ the excess iron in our CuFeAs samples is-according to our super-cell calculation-in a localized state which provides a ferromagnetic response upon an external field. Still, not all secondary iron show this ferromagnetism, a critical local iron concentration seems to be crucial for the local moment formation.

Our theoretical analysis draws attention to the important effect of charge doping that evolves from total $\mathrm{Cu}$ deficiency and $\mathrm{Fe} / \mathrm{Cu}$ substitution. The $\mathrm{Cu}$ deficiency alone corresponds to hole doping and stabilizes a magnetic ground state of the FeAs layer with a moment of $\approx 0.4 \mu_{B}$, which is in excellent agreement with the results of Qian et al. [24].

In contrast to the effective electron doping by excess iron in FeTe, LiOHFeSe [64], or LiFeAs [62] the secondary iron in $\mathrm{CuFeAs}$ acts as a hole donor which drives the original Fe layers close to the magnetic instability. This effect is enhanced by local polarization due to hybridization of secondary Fe with $\mathrm{Fe}$ atoms in the adjacent Fe-As layer and the rather large magnetic moment of the neighboring secondary Fe. However, $84 \%$ of regular $\mathrm{Fe}$ atoms have no or only one secondary Fe neighbor (according to binomial distribution). That means that the vast majority of regular Fe atoms is only weakly influenced by this local effect. These theoretical results allow for the following conclusion: depending on the secondary iron concentration the regular iron sites in $\mathrm{CuFeAs}$ have either an ordered magnetic moment of $\approx 0.4 \mu_{B}$ or are paramagnetic (ground state of stoichiometric CuFeAs), both in agreement with high field Mössbauer spectroscopy. From the Mössbauer perspective the partial presence of small magnetic moments at the regular $\mathrm{Fe}$ sites is probable.

So far the interpretation suggests that both small magnetic moments $\left(\approx 0.4 \mu_{B}\right)$ at the regular site and large moments $\left(\gtrsim 1 \mu_{B}\right)$ at the secondary $2 c$ site are present. We attribute the saturation moment of $\approx 0.2 \mu_{B} / \mathrm{Fe}$ seen in magnetization measurements mainly to the ferromagnetic secondary iron. In that sense that number corresponds to $\approx 2 \mu_{B} / \mathrm{Fe}_{2 n d}$, which lies between the values obtained in Mössbauer spectroscopy $\left(\approx 1 \mu_{B}\right)$ and predicted theoretically $\left(2.5 \mu_{B}\right)$. In $\mu$ SR the transverse damping rate of $\lambda_{T}=65 \mu \mathrm{s}^{-1}$ corresponds to a characteristic field of $76 \mathrm{mT}$. This is the characteristic field expected from a stripe AFM (typically observed in iron arsenides) order with $0.4 \mu_{B}$ magnetic moments. On the other hand $1.2 \mu_{B}$ secondary iron moments cause the same fields at the traditional muon site $0.6 \AA$ above the iron plane. In that sense the $\mu \mathrm{SR}$ data are consistent with both explanations of magnetism. Furthermore, the two contributions may be the reason why a two component fit works for the magnetic volume fraction $f_{\text {mag }}$ in $\mu$ SR (Fig. 7). In Mössbauer spectroscopy the signature of two origins of magnetism is the temperature dependence of $B_{\text {tot }}$ (Fig. 2) which shows almost a plateau between $8 \mathrm{~K}$ and $15 \mathrm{~K}$ : The increase above $15 \mathrm{~K}$ is obviously related to transferred hyperfine field of the secondary iron moments, whereas below $8 \mathrm{~K}$ the AFM order of regular iron starts. Transferred hyperfine fields are probably responsible for the $0.15 \mathrm{~T}$ larger $B_{\text {hyp }}$ observed in the $\mu \mathrm{SR}$ sample, where the excess iron content is enhanced (Sec. III F) compared to the neutron sample.

The coupling of regular and secondary Fe shown in our calculation may explain why both iron sites order at roughly the same temperature of $11 \mathrm{~K}$ in $\mathrm{CuFeAs}$. We note that this transition temperature is comparable to $\left(\mathrm{Li}_{1-y} \mathrm{Fe}_{\mathrm{y}} \mathrm{OH}\right) \mathrm{FeSe}$ $[65,66]$ : In that compound ferromagnetism occurs at a temperature of $10 \mathrm{~K}$ from a randomly distributed, small amount of secondary iron at interlayer position as well. The importance of the coupling of regular and secondary iron sites can be adopted to the structurally related $\mathrm{CuFeSb}$, where both the ( $\mathrm{Sb}, \mathrm{Fe}$ ) mixing and a strong coupling of the magnetic order parameters (Fig. 15) are easily observed in Mössbauer spectroscopy.

The strong coupling of the magnetic order parameters of the regular $\mathrm{Fe}$ site and the secondary site in a 111 
compound is the salient experimental result of the $\mathrm{CuFeSb}$ investigations. We were able to reveal the two-dimensional character of magnetic interactions and the alignment of soft ferromagnetic moments in the $a b$ plane. The magnetic order parameter shows a smooth temperature dependence and thus excludes a steplike behavior, which had come into consideration in neutron measurements [24]. The conversion ratio of Mössbauer hyperfine field and magnetic moment determined from neutron diffraction is $-10 \mathrm{~T} / \mu_{B}$. This is less than the typical value of iron arsenides/chalcogenides [40], approaching the value of $-13 \mathrm{~T} / \mu_{B}$ for a predominant core contribution [67], which is typically observed in more localized systems. The localization-or reduced covalencycaused by the large Fe-Sb bond distance of $2.69 \AA$ [24] becomes apparent with regard to the Mössbauer isomer shift $\delta(T \rightarrow 0)=0.63 \mathrm{~mm} / \mathrm{s}$ with respect to room temperature iron, which is $0.1 \mathrm{~mm} / \mathrm{s}$ more than CuFeAs, amongst many other iron arsenides. The difference in magnitude $(60 \%)$ of the iron magnetic moment at the regular site and at the $2 c$ site is in qualitative agreement with the supercell calculation for CuFeAs. Because of the more localized character of the regular iron site a classical explanation in terms of crystal field seems applicable: The regular iron site corresponds to a tetrahedral site with strong bonding to the Sb ions and thus a strong crystal field, which favors an intermediate spin state, whereas the iron at the copper site in octahedral symmetry might only sense small crystal field splitting and thus a high spin state might be preferred.

$\mathrm{CuFeSb}$ was known to support the unified picture of anion height dependent magnetism [18,20]. Due to ambiguous crystallographic and magnetic data such a statement was questionable for $\mathrm{CuFeAs}$ so far. Our neutron diffraction data now show that the structural parameters of CuFeAs (Table I, $h_{\text {anion }}=1.53 \AA, \alpha_{2 \times}=101.3^{\circ}$ ) are close to those of LiFeAs $[1,68]\left(h_{\text {anion }}=1.51 \AA, \alpha_{2 \times}=103.1^{\circ}\right)$. Both structural data and the crucial role of $h_{\text {anion }}$ for magnetism are confirmed by our theoretical calculations. The rather small anion height disfavors the formation of a large $\mathrm{Fe}$ moment for the regular Fe site in the Fe-As layer. Our experimental data are inconsistent with ferromagnetism at the regular iron site. Eventually, CuFeAs follows the principles of anion height driven magnetism. Beyond that, our investigation unveils that a proper determination of structural data and stoichiometry is fundamental for the understanding of divergent behavior of imperfect samples. In our case $(\mathrm{Cu}, \mathrm{Fe})$ substitution occurs in both batches in the same quantity, causing localized ferromagnetic secondary $\mathrm{Fe}$, eventually changing the ground state from a paramagnetic to an AFM ordered one via hole doping, and finally prohibiting long range order.

\section{SUMMARY}

We investigated the magnetism of $\mathrm{CuFeAs}$ and $\mathrm{CuFeSb}$ using microscopic local probe techniques and theoretical calculations. In both compounds $\approx 10 \%$ of the $\mathrm{Cu}$ sites are occupied by Fe. In both compounds these secondary iron atoms carry larger moments than the regular site, while both order parameters are strongly coupled. The large anion height in $\mathrm{CuFeSb}$ is consistent with the ferromagnetic ground state with a large moment. In CuFeAs short-range order of weak magnetic moments $\left(<0.4 \mu_{\mathrm{B}}\right)$ at the regular site is observed below $11 \mathrm{~K}$. This is consistent with our DFT calculation predicting a magnetic instability of $\mathrm{CuFeAs}$ caused by a relatively small arsenic height of $\approx 1.5 \AA$, and enhanced by the hole doping effect of secondary iron.

\section{ACKNOWLEDGMENTS}

We appreciate the financial support by the Deutsche Forschungsgemeinschaft (DFG) through the priority program SPP 1458 (KL 1086/10-2) and the Research Training Group GRK 1621. The identification of any commercial product or trade name does not imply endorsement or recommendation by the National Institute of Standards and Technology.
[1] J. H. Tapp, Z. Tang, B. Lv, K. Sasmal, B. Lorenz, P. C. W. Chu, and A. M. Guloy, Phys. Rev. B 78, 060505(R) (2008).

[2] Y. Kamihara, H. Hiramatsu, M. Hirano, R. Kawamura, H. Yanagi, T. Kamiya, and H. Hosono, J. Am. Chem. Soc. 128, 10012 (2006).

[3] C. Chu, F. Chen, M. Gooch, A. Guloy, B. Lorenz, B. Lv, K. Sasmal, Z. Tang, J. Tapp, and Y. Xue, Physica C 469, 326 (2009).

[4] Z. Deng, X. Wang, Q. Liu, S. Zhang, Y. Lv, J. Zhu, R. Yu, and C. Jin, EPL (Europhys. Lett.) 87, 37004 (2009).

[5] K. Hashimoto, S. Kasahara, R. Katsumata, Y. Mizukami, M. Yamashita, H. Ikeda, T. Terashima, A. Carrington, Y. Matsuda, and T. Shibauchi, Phys. Rev. Lett. 108, 047003 (2012)

[6] P. D. Johnson, G. Xu, and W.-G. Yin, Iron-Based Superconductivity, Vol. 211 (Springer, Cham, Heidelberg, 2015).

[7] J. Wright, T. Lancaster, I. Franke, A. Steele, J. Möller, M. Pitcher, A. Corkett, D. Parker, D. Free, F. Pratt et al., Phys. Rev. B 85, 054503 (2012).
[8] F. Steckel, R. Beck, M. Roslova, M. Abdel-Hafiez, A. U. Wolter, I. Morozov, S. Wurmehl, C. Hess, and B. Büchner, J. Supercond. Nov. Magn. 28, 1123 (2015).

[9] P. Nag, R. Schlegel, D. Baumann, H.-J. Grafe, R. Beck, S. Wurmehl, B. Büchner, and C. Hess, Sci. Rep. 6, 27926 (2016).

[10] S. Chi, R. Aluru, U. R. Singh, R. Liang, W. N. Hardy, D. Bonn, A. Kreisel, B. M. Andersen, R. Nelson, T. Berlijn et al., Phys. Rev. B 94, 134515 (2016).

[11] S. Borisenko, V. Zabolotnyy, D. Evtushinsky, T. Kim, I. Morozov, A. Yaresko, A. Kordyuk, G. Behr, A. Vasiliev, R. Follath et al., Phys. Rev. Lett. 105, 067002 (2010).

[12] J. Ferber, K. Foyevtsova, R. Valentí, and H. O. Jeschke, Phys. Rev. B 85, 094505 (2012).

[13] T. Miyake, K. Nakamura, R. Arita, and M. Imada, J. Phys. Soc. Jpn. 79, 044705 (2010).

[14] L. Ma, J. Zhang, G. F. Chen, and W. Yu, Phys. Rev. B 82, 180501(R) (2010).

[15] D. J. Singh, Phys. Rev. B 78, 094511 (2008). 
[16] Y.-F. Li and B.-G. Liu, Eur. Phys. J. B 72, 153 (2009).

[17] C.-Y. Moon, S. Y. Park, and H. J. Choi, Phys. Rev. B 80, 054522 (2009).

[18] C.-Y. Moon and H. J. Choi, Phys. Rev. Lett. 104, 057003 (2010).

[19] S. Li, C. de La Cruz, Q. Huang, Y. Chen, J. W. Lynn, J. Hu, Y.-L. Huang, F.-C. Hsu, K.-W. Yeh, M.-K. Wu, and P. Dai, Phys. Rev. B 79, 054503 (2009).

[20] W.-G. Yin, C.-C. Lee, and W. Ku, Phys. Rev. Lett. 105, 107004 (2010).

[21] Y. Mizuguchi, Y. Hara, K. Deguchi, S. Tsuda, T. Yamaguchi, K. Takeda, H. Kotegawa, H. Tou, and Y. Takano, Supercond. Sci. Technol. 23, 054013 (2010).

[22] E. E. Rodriguez, C. Stock, P. Zajdel, K. L. Krycka, C. F. Majkrzak, P. Zavalij, and M. A. Green, Phys. Rev. B 84, 064403 (2011).

[23] H. Yanagi, R. Kawamura, T. Kamiya, Y. Kamihara, M. Hirano, T. Nakamura, H. Osawa, and H. Hosono, Phys. Rev. B 77, 224431 (2008).

[24] B. Qian, J. Lee, J. Hu, G. C. Wang, P. Kumar, M. H. Fang, T. J. Liu, D. Fobes, H. Pham, L. Spinu, X. S. Wu, M. Green, S. H. Lee, and Z. Q. Mao, Phys. Rev. B 85, 144427 (2012).

[25] A. Sirohi, C. K. Singh, G. S. Thakur, P. Saha, S. Gayen, A. Gaurav, S. Jyotsna, Z. Haque, L. C. Gupta, M. Kabir et al., Appl. Phys. Lett. 108, 242411 (2016).

[26] G. S. Thakur, Z. Haque, L. C. Gupta, and A. K. Ganguli, J. Phys. Soc. Jpn. 83, 054706 (2014).

[27] B. Qian, J. Hu, J. Liu, Z. Han, P. Zhang, L. Guo, X. Jiang, T. Zou, M. Zhu, C. R. Dela Cruz, X. Ke, and Z. Q. Mao, Phys. Rev. B 91, 014504 (2015).

[28] G. Wang, X. Shi, and D. Wang, J. Alloys Compd. 686, 38 (2016).

[29] S. Kamusella and H.-H. Klauss, Hyperfine Interact. 237, 1 (2016).

[30] J. Lynn, Y. Chen, S. Chang, Y. Zhao, S. Chi, W. Ratcliff, B. Ueland, R. Erwin et al., J. Res. NIST 117, 61 (2012).

[31] J. Rodríguez-Carvajal, Physica B 192, 55 (1993).

[32] J. L. Pouchou and F. Pichoir, Rech. Aerosp. 3, 167 (1984).

[33] K. Koepernik and H. Eschrig, Phys. Rev. B 59, 1743 (1999).

[34] K. Koepernik, B. Velický, R. Hayn, and H. Eschrig, Phys. Rev. B 55, 5717 (1997).

[35] J. P. Perdew and Y. Wang, Phys. Rev. B 45, 13244 (1992).

[36] J. P. Perdew, K. Burke, and M. Ernzerhof, Phys. Rev. Lett. 77, 3865 (1996).

[37] D. Kasinathan, A. Ormeci, K. Koch, U. Burkhardt, W. Schnelle, A. Leithe-Jasper, and H. Rosner, New J. Phys. 11, 025023 (2009).

[38] M. Schmitt, J. Málek, S.-L. Drechsler, and H. Rosner, Phys. Rev. B 80, 205111 (2009).

[39] J. P. Wright, J. P. Attfield, and P. G. Radaelli, Phys. Rev. B 66, 214422 (2002).

[40] S. Kamusella, K. To Lai, L. Harnagea, R. Beck, U. Pachmayr, G. S. Thakur, and H.-H. Klauss, Phys. Status Solidi B 254 (2017).

[41] S. Kitao, Y. Kobayashi, S. Higashitaniguchi, M. Kurokuzu, M. Saito, T. Mitsui, Y. Kamihara, M. Hirano, H. Hosono, and M. Seto, J. Phys.: Conf. Ser. 217, 012120 (2010).

[42] I. Dézsi, C. Fetzer, A. Gombkötô, I. Szúcs, J. Gubicza, and T. Ungár, J. Appl. Phys. 103, 104312 (2008).

[43] M. Yuzuri, R. Tahara, and Y. Nakamura, J. Phys. Soc. Jpn. 48, 1937 (1980).

[44] D. Rancourt, J. Daniels, and H. Lam, Can. J. Phys. 63, 1540 (1985).
[45] C. Bernhard, C. Wang, L. Nuccio, L. Schulz, O. Zaharko, J. Larsen, C. Aristizabal, M. Willis, A. Drew, G. Varma et al., Phys. Rev. B 86, 184509 (2012).

[46] C. N. Wang, P. Marsik, R. Schuster, A. Dubroka, M. Rössle, C. Niedermayer, G. Varma, A. Wang, X. Chen, T. Wolf et al., Phys. Rev. B 85, 214503 (2012).

[47] J. D. Wright, M. J. Pitcher, W. Trevelyan-Thomas, T. Lancaster, P. J. Baker, F. L. Pratt, S. J. Clarke, and S. J. Blundell, Phys. Rev. B 88, 060401(R) (2013).

[48] L. Ma, G. F. Chen, D.-X. Yao, J. Zhang, S. Zhang, T.-L. Xia, and W. Yu, Phys. Rev. B 83, 132501 (2011).

[49] I. Presniakov, I. Morozov, A. Sobolev, M. Roslova, A. Boltalin, O. Volkova, and A. Vasilev, JETP Lett. 97, 583 (2013).

[50] A. Yaouanc and P. D. De Réotier, Muon Spin Rotation, Relaxation, and Resonance: Applications to Condensed Matter, Vol. 147 (Oxford University Press, New York, 2011).

[51] F. Pratt, J. Phys.: Condens. Matter 19, 456207 (2007).

[52] L. Pauwels, G. Maervoet, and R. Vervaeke, Zeitschrift für anorganische und allgemeine Chemie 397, 307 (1973).

[53] I. I. Mazin, M. D. Johannes, L. Boeri, K. Koepernik, and D. J. Singh, Phys. Rev. B 78, 085104 (2008).

[54] T. Goltz, V. Zinth, D. Johrendt, H. Rosner, G. Pascua, H Luetkens, P. Materne, and H.-H. Klauss, Phys. Rev. B 89, 144511 (2014).

[55] A. Leithe-Jasper, W. Schnelle, C. Geibel, and H. Rosner, Phys. Rev. Lett. 101, 207004 (2008).

[56] Although the Stoner criterion is designed for the description of ferromagnetic states, it is in many cases a good indicator for the instability toward any kind of band magnetism, see for instance Ref. [54].

[57] Due to the large crystallographic cell and low symmetry for the simulation of the respective experimental Fe excess, the calculations converge only badly. Thus we restricted ourselves to $25 \% \mathrm{Fe}$ excess.

[58] K. Yamaguchi, H. Yamamoto, Y. Yamaguchi, and H. Watanabe, J. Phys. Soc. Jpn. 33, 1292 (1972).

[59] R. Kumar, K. S. Harchand, Vishwamittar K. Chandra, P. Jernberg, T. Ericsson, R. Wappling, Phys. Rev. B 32, 69 (1985).

[60] J. Knudsen and S. Mørup, Le Journal de Physique Colloques 41, C1-155 (1980).

[61] X. Wang, Q. Liu, Y. Lv, W. Gao, L. Yang, R. Yu, F. Li, and C. Jin, Solid State Commun. 148, 538 (2008).

[62] M. J. Pitcher, T. Lancaster, J. D. Wright, I. Franke, A. J. Steele, P. J. Baker, F. L. Pratt, W. T. Thomas, D. R. Parker, S. J. Blundell et al., J. Am. Chem. Soc. 132, 10467 (2010).

[63] S. Li, C. de la Cruz, Q. Huang, G. F. Chen, T.-L. Xia, J. L. Luo, N. L. Wang, and P. Dai, Phys. Rev. B 80, 020504 (2009).

[64] W. Chen, C. Zeng, E. Kaxiras, and Z. Zhang, Phys. Rev. B 93, 064517 (2016).

[65] U. Pachmayr, F. Nitsche, H. Luetkens, S. Kamusella, F. Brückner, R. Sarkar, H.-H. Klauss, and D. Johrendt, Angew. Chem., Int. Ed. 54, 293 (2015).

[66] J. W. Lynn, X. Zhou, C. K. H. Borg, S. R. Saha, J. Paglione, and E. E. Rodriguez, Phys. Rev. B 92, 060510(R) (2015).

[67] O. Eriksson and A. Svane, J. Phys.: Condens. Matter 1, 1589 (1989).

[68] M. J. Pitcher, D. R. Parker, P. Adamson, S. J. C. Herkelrath, A. T. Boothroyd, R. M. Ibberson, M. Brunelli, and S. J. Clarke, Chem. Commun. 45, 5918 (2008). 